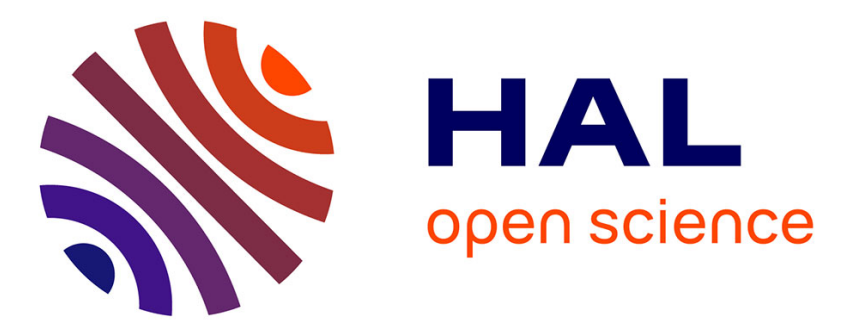

\title{
Analysis of a simplified model of rigid structure floating in a viscous fluid
}

Debayan Maity, Jorge San Martin, Takéo Takahashi, Marius Tucsnak

\section{To cite this version:}

Debayan Maity, Jorge San Martin, Takéo Takahashi, Marius Tucsnak. Analysis of a simplified model of rigid structure floating in a viscous fluid. Journal of Nonlinear Science, 2019, 29 (5), pp.1975-2020. 10.1007/s00332-019-09536-5 . hal-01889892

\section{HAL Id: hal-01889892 \\ https://hal.science/hal-01889892}

Submitted on 8 Oct 2018

HAL is a multi-disciplinary open access archive for the deposit and dissemination of scientific research documents, whether they are published or not. The documents may come from teaching and research institutions in France or abroad, or from public or private research centers.
L'archive ouverte pluridisciplinaire HAL, est destinée au dépôt et à la diffusion de documents scientifiques de niveau recherche, publiés ou non, émanant des établissements d'enseignement et de recherche français ou étrangers, des laboratoires publics ou privés. 


\title{
Analysis of a simplified model of rigid structure floating in a viscous fluid.
}

\author{
Debayan Maity ${ }^{\dagger 1}$, Jorge San Martín², Takéo Takahashi*3, and Marius Tucsnak ${ }^{\dagger 1}$ \\ ${ }^{1}$ Institut de Mathématiques, Université de Bordeaux, Bordeaux INP, CNRS, \\ F-33400 Talence, France \\ ${ }^{2}$ DIM, Universidad de Chile, Blanco Encalada, Chile \\ ${ }^{3}$ Université de Lorraine, CNRS, Inria, IECL, F-54000 Nancy, France \\ debayan.maity@u-bordeaux.fr,jorge@dim.u-chile.cl,takeo.takahashi@inria.fr, \\ marius.tucsnak@u-bordeaux.fr
}

October 8, 2018

\begin{abstract}
We study the interaction of surface water waves with a floating solid constraint to move only in the vertical direction. The first novelty we bring in is that we propose a new model for this interaction, taking into consideration the viscosity of the fluid. This is done supposing that the flow obeys a shallow water regime (modeled by the viscous Saint-Venant equations in one space dimension) and using a Hamiltonian formalism. Another contribution of this work is establishing the well-posedness of the obtained PDEs/ODEs system in function spaces similar to the standard ones for strong solutions of viscous shallow water equations. Our wellposedness results are local in time for every initial data and global in time if the initial data are close (in appropriate norms) to an equilibrium state. Moreover, we show that the linearization of our system around an equilibrium state can be described, at least for some initial data, by an integro-fractional differential equation related to the classical Cummins equation and which reduces to the Cummins equation when the viscosity vanishes and the fluid is supposed to fill the whole space. Finally, we describe some numerical tests, performed on the original nonlinear system, which illustrate the return to equilibrium and the influence of the viscosity coefficient.
\end{abstract}

Key words. Viscous shallow water equations, floating structure, fluid-structure interaction, strong solutions, return to equilibrium.

AMS subject classifications. 35Q35, 74F10.

\section{Contents}

1 Introduction

${ }^{*}$ Takéo Takahashi acknowledges the support of the Agence Nationale de la Recherche, project IFSMACS, ANR15-CE40-0010.

${ }^{\dagger}$ Debayan Maity and Marius Tucsnak acknowledge the support of the Agence Nationale de la Recherche - Deutsche Forschungsgemeinschaft (ANR - DFG), project INFIDHEM, ID ANR-16-CE92-0028. 
6 Global in time existence and uniqueness 16

6.1 Linearization around a stationary state . . . . . . . . . . . . . . . . . . 16

6.2 Study of the Linear Problem . . . . . . . . . . . . . . . . . . . 19

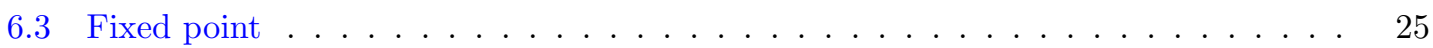

$\begin{array}{llr}7 & \text { Return to the Equilibrium } & 27\end{array}$

8 Numerical Simulations $\quad 32$

\section{Introduction}

The motion of rigid bodies in a fluid is a widely studied subject in both engineering and mathematical literature. This is due to important applications (naval and aerospace engineering, biology, medicine,...$)$ and to the mathematical challenges, namely the existence of free boundaries, raised by the corresponding mathematical models. The study of the case in which the rigid bodies are completely immersed in the fluid goes back to Euler, Kelvin and Kirchhoff. Due to the important number of works (namely in the last two decades) devoted to this subject, the corresponding mathematical questions may be considered by now well understood, for various types of fluids (ideal, viscous incompressible, compressible, ... ). We refer, for instance, to [4] and references therein for a concise description of recent progress in this field. The case when the solids are floating, thus only partially immersed and interacting with the water waves, has been much less studied in the literature, essentially in the case of an ideal fluid. We refer to the classical work of John $[11,12]$ or to the monograph Falnes [7] for the linearized theory and to the review paper of Lannes [14] for the description of recent progress in the field. As far as we know, the case of a viscous fluid has not been tackled, at least from a mathematical view point. The aim of this work is to partially fill this gap by considering a PDE system modeling the coupled motion of a free surface viscous fluid and of a solid, constrained to move in the vertical direction only, which is floating on this free surface. The main contributions of this work are:

- Proposing, via a Hamiltonian formalism, a new mathematical model which takes into account the viscosity of the fluid, for the coupled of a free boundary shallow fluid and of a rigid body floating on the surface.

- Proving the existence and uniqueness of strong solutions (locally in time or globally in time for small data).

- Proposing a reduced model and performing numerical simulations in the linear case.

The content of the subsequent sections is outlined below. In Section 2 we derive the governing equations using a Hamiltonian formalism. In Section 3 we show that our model is energetically consistent and we state our main existence and uniqueness results. In Section 4 we write the governing equation in an equivalent form obtained by "eliminating" the unknown functions involving the floating solid. Section 5 is devoted to the proof of the local in time existence and uniqueness result, whereas in Section 6 we prove global in time existence and uniqueness of solutions for initial data which are close to an equilibrium state. Finally, in Section 7 we analyze the return to equilibrium problem and in Section 8, we present some numerical simulations for this problem. 


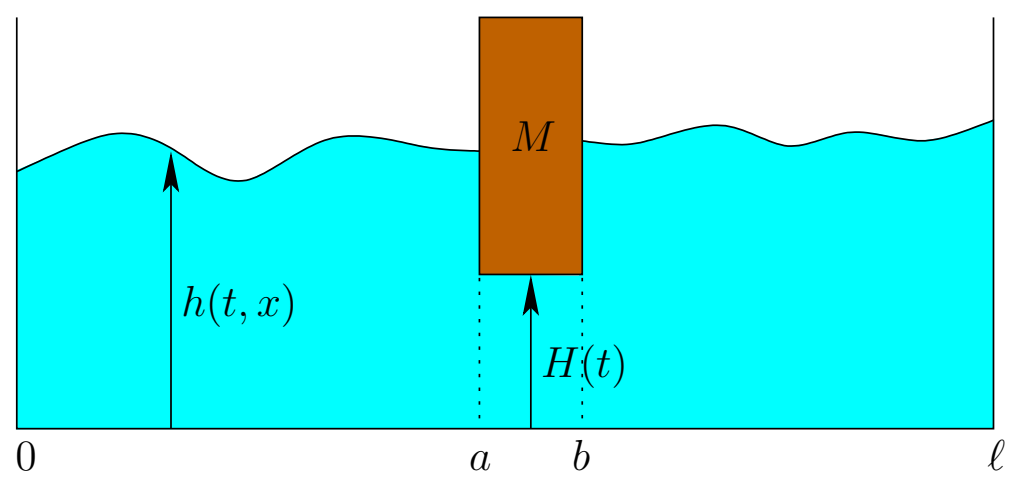

Figure 1: Configuration

\section{Modelling}

In this section, we derive, using a Hamiltonian formalism, the simplified model which is analyzed in the remaining part of this work. The fluid is described using the viscous Saint Venant equations, whereas the solid obeys Newton's second law. To couple the two models we use a Hamiltonian formalism, passing by the following steps:

- Introduce the total energy of the fluid-floating object system within the Saint-Venant approximation and define conservation of mass as a constraint, following Petit and Rouchon [17].

- Use of a Hamiltonian formalism to derive the governing equations, by combining the approach introduced in [17] for the inviscid case with the methodology used in Gay-Balmaz and Yoshimura [8] in the case the Navier-Stokes-Fourier system, which allows us to include the viscous effects in the system.

To describe our model we need some notation, which is introduced below. Denote respectively by $\rho(t, x), v(t, x)$ and $h(t, x)$ the density, the velocity, and the height of the free surface of the fluid (see Figure 1). These quantities depend on the time $t \geqslant 0$ and on the position $x \in[0, \ell]$. We also denote by $H(t)$ the height of the cylinder. The mass $M$ of the rigid body is a positive constant. We also assume that the cylinder moves only vertically and we set

$$
\mathcal{I}:=[a, b]:=\text { the projection of the cylinder on the flat bottom, } \mathcal{E}:=(0, \ell) \backslash \mathcal{I},
$$

with $\overline{\mathcal{I}} \subset(0, \ell)$.

The function $H$, which depends only on time is supposed to satisfy

$$
0<H(t)<\min \left(h\left(t, a^{-}\right), h\left(t, b^{+}\right)\right) \quad(t \geqslant 0),
$$

so that the cylinder is immersed into the fluid and does not touch the bottom.

We are now on a position to derive the governing equations. The first one is the equation of mass conservation:

$$
\frac{\partial h}{\partial t}+\frac{\partial(h v)}{\partial x}=0 \quad(t>0, x \in[0, \ell]) .
$$

For $x \in \mathcal{I}$ the height of fluid is given by the position of the cylinder, i.e.:

$$
h(t, x)=H(t) \quad(t>0, x \in \mathcal{I}) .
$$

Equations (2.2) and (2.3) appear below as constraints in our Hamiltonian formalism. 
In order to derive the other equations, we introduce the kinetic energy $K_{f}$ and the potential energy $U_{f}$ of the fluid:

$$
\begin{aligned}
K_{f} & =\frac{1}{2} \int_{(0, \ell)} \rho h v^{2} \mathrm{~d} x, \\
U_{f} & =\int_{(0, \ell)}\left[\frac{1}{2} \rho g h^{2}+e(s(t, x))\right] \mathrm{d} x,
\end{aligned}
$$

where $g$ is the gravity acceleration, and $e(s)$ represents the internal energy density which is a function of the density of entropy $s$. We assume that the fluid is homogeneous and incompressible, so that that its density $\rho$ is a constant.

We also introduce the kinetic energy $K_{f}$ and the potential energy $U_{f}$ of the solid:

$$
K_{s}=\frac{1}{2} M \dot{H}^{2} \quad U_{s}=M g H .
$$

We obtain below the governing equations by writing the stationarity for the total action of the system under the constraints (2.2) and (2.3). To this aim, it seems more convenient, in particular to treat the continuity restriction (2.2), to introduce the variable $q$ defined by

$$
q(t, x)=h(t, x) v(t, x) \quad(t>0, x \in[0, \ell]) .
$$

The total action of the system is given by

$$
\begin{aligned}
\mathcal{A}(h, v, H) & =\left(K_{f}+K_{s}\right)-\left(U_{f}+U_{s}\right) \\
& =\int_{0}^{T}\left\{\int_{0}^{\ell}\left[\frac{1}{2} \rho h v^{2}-\frac{1}{2} \rho g h^{2}-e(s(t, x))\right] \mathrm{d} x+\frac{1}{2} M \dot{H}^{2}-M g H\right\} \mathrm{d} t .
\end{aligned}
$$

In order to include the restrictions (2.2)-(2.3), we introduce two Lagrange multipliers:

$$
\begin{array}{lr}
\lambda_{1}(t, x) & (t \geqslant 0, x \in[0, \ell]), \\
\lambda_{2}(t, x) & (t \geqslant 0, x \in \mathcal{I}) .
\end{array}
$$

With the above notation, the governing equations are obtained as stationarity conditions for the Lagrangian defined by

$$
\begin{aligned}
\mathcal{L}\left(h, q, H, \lambda_{1}, \lambda_{2}\right)=\int_{0}^{T}\left\{\int _ { 0 } ^ { \ell } \left[\frac{1}{2} \rho \frac{q^{2}}{h}-\frac{1}{2} \rho g h^{2}\right.\right. & \left.+\rho \lambda_{1}\left(\frac{\partial h}{\partial t}+\frac{\partial q}{\partial x}\right)-e(s(t, x))\right] \mathrm{d} x \\
& \left.+\int_{\mathcal{I}}\left[\lambda_{2}(H-h)\right] \mathrm{d} x+\frac{1}{2} M \dot{H}^{2}-M g H\right\} \mathrm{d} t .
\end{aligned}
$$

To achieve this aim, we consider virtual displacements of the trajectory, given by the independent variations $\delta h(x, t), \delta H(t)$ and $\delta q$. Since $q$ is a velocity type quantity, whereas $h$ and $H$ are displacements, we introduce the function $\varphi$ and its variation $\delta \varphi$ defined by

$$
\frac{\partial \varphi}{\partial t}=q \quad \text { and } \quad \frac{\partial(\delta \varphi)}{\partial t}=\delta q .
$$

The governing equations are obtained by imposing that $\delta \mathcal{L}=0$ for any virtual displacement $\delta h(t, x), \delta H(t)$ and $\delta \varphi(t, x)$ such that

$$
\begin{gathered}
\delta h(0, x)=\delta h(T, x)=\delta H(0)=\delta H(T)=\delta \varphi(0, x)=\delta \varphi(T, x)=0, \\
\delta \varphi(t, 0)=\delta \varphi(t, \ell)=0 .
\end{gathered}
$$


Moreover, to take in consideration friction forces inside the fluid, we follow the approach in [8] by assuming that the entropy trajectory $s$ and its variation $\delta s$ satisfy the variational constraint

$$
\frac{\partial e}{\partial s} \delta s=\frac{\mu}{h} \frac{\partial q}{\partial x} \frac{\partial(\delta \varphi)}{\partial x}
$$

where the constant $\mu>0$ is the viscosity of the fluid, together with the phenomenological constraint

$$
\frac{\partial e}{\partial s} \dot{s}=\frac{\mu}{h}\left(\frac{\partial q}{\partial x}\right)^{2} .
$$

Note that the above expression of this friction term leads to an nonstandard form of the viscous Saint-Venant equations, see Remark 2.1 below.

Using (2.5) we get

$$
\begin{aligned}
\delta \mathcal{L}=\int_{0}^{T}\left\{\int _ { 0 } ^ { \ell } \left[\rho \frac{q}{h}(\delta q)-\frac{1}{2} \rho \frac{q^{2}}{h^{2}}(\delta h)-\right.\right. & \left.\left.\rho g h(\delta h)+\rho \lambda_{1}\left(\frac{\partial(\delta h)}{\partial t}+\frac{\partial(\delta q)}{\partial x}\right)\right]-\frac{\partial e}{\partial s}(\delta s)\right) \mathrm{d} x \\
& \left.+\int_{\mathcal{I}}\left[\lambda_{2}(\delta H-\delta h)\right] \mathrm{d} x+M \dot{H}(\delta \dot{H})-M g(\delta H)\right\} \mathrm{d} t .
\end{aligned}
$$

The above formula and (2.6) yield:

$$
\begin{array}{r}
\delta \mathcal{L}=\int_{0}^{T}\left\{\int_{0}^{\ell}\left[\rho \frac{q}{h} \frac{\partial(\delta \varphi)}{\partial t}-\frac{1}{2} \rho \frac{q^{2}}{h^{2}}(\delta h)-\rho g h(\delta h)+\rho \lambda_{1}\left(\frac{\partial(\delta h)}{\partial t}+\frac{\partial}{\partial x} \frac{\partial(\delta \varphi)}{\partial t}\right)\right]-\frac{\partial e}{\partial s}(\delta s)\right) \mathrm{d} x \\
\left.+\int_{\mathcal{I}}\left[\lambda_{2}((\delta H)-(\delta h))\right] \mathrm{d} x+M \dot{H}(\delta \dot{H})-M g(\delta H)\right\} \mathrm{d} t .
\end{array}
$$

Considering (2.7) and integrating by parts in time the formula (2.10), we find

$$
\begin{aligned}
\delta \mathcal{A}=\int_{0}^{T}\left\{\int_{0}^{\ell}[-\right. & \left.\rho \frac{\partial}{\partial t}\left(\frac{q}{h}\right) \delta \varphi-\rho \frac{\partial \lambda_{1}}{\partial t} \frac{\partial}{\partial x} \delta \varphi-\frac{\partial e}{\partial s} \delta s\right] \mathrm{d} x \\
& +\int_{0}^{\ell}\left[\left(-\frac{1}{2} \rho \frac{q^{2}}{h^{2}}-\rho g h-\rho \frac{\partial \lambda_{1}}{\partial t}\right) \delta h\right] \mathrm{d} x-\int_{\mathcal{I}}\left[\lambda_{2} \delta h\right] \mathrm{d} x \\
& \left.+\left(\int_{\mathcal{I}} \lambda_{2} \mathrm{~d} x-M \ddot{H}-M g\right) \delta H\right\} \mathrm{d} t .
\end{aligned}
$$

Using the model (2.9) in (2.11) we get

$$
\begin{aligned}
& \delta \mathcal{A}=\int_{0}^{T}\left\{\int_{0}^{\ell}\left[-\rho \frac{\partial}{\partial t}\left(\frac{q}{h}\right)(\delta \varphi)-\rho \frac{\partial \lambda_{1}}{\partial t} \frac{\partial}{\partial x}(\delta \varphi)-\mu \frac{1}{h} \frac{\partial q}{\partial x} \frac{\partial(\delta \varphi)}{\partial x}\right] \mathrm{d} x\right. \\
& +\int_{0}^{\ell}\left[\left(-\frac{1}{2} \rho \frac{q^{2}}{h^{2}}-\rho g h-\rho \frac{\partial \lambda_{1}}{\partial t}\right)(\delta h)\right] \mathrm{d} x-\int_{a}^{b}\left[\lambda_{2}(\delta h)\right] \mathrm{d} x \\
& \left.+\left(\int_{a}^{b} \lambda_{2} \mathrm{~d} x-M \ddot{H}-M g\right)(\delta H)\right\} \mathrm{d} t .
\end{aligned}
$$

Now we integrate by parts in space but we need to take care about the fact that some quantities can present discontinuities at the interfaces solid-liquid. We thus introduce the notation

$$
[f]_{a}:=f\left(a^{+}\right)-f\left(a^{-}\right), \quad[f]_{b}:=f\left(b^{+}\right)-f\left(b^{-}\right)
$$


and we use the following formula for the integration by parts with discontinuities:

$$
\int_{0}^{\ell} u v^{\prime} d x=-\int_{0}^{\ell} u^{\prime} v d x-[u v]_{a}-[u v]_{b}
$$

We thus obtain

$$
\begin{aligned}
\delta \mathcal{A}=\int_{0}^{T}\left\{\int_{0}^{\ell}[\right. & \left.-\rho \frac{\partial}{\partial t}\left(\frac{q}{h}\right)+\rho \frac{\partial}{\partial x}\left(\frac{\partial \lambda_{1}}{\partial t}\right)+\frac{\partial}{\partial x}\left(\frac{\mu}{h} \frac{\partial q}{\partial x}\right)\right](\delta \varphi) \mathrm{d} x \\
& +\left[\rho \frac{\partial \lambda_{1}}{\partial t}+\frac{\mu}{h} \frac{\partial q}{\partial x}\right]_{a}(\delta \varphi)(t, a)+\left[\rho \frac{\partial \lambda_{1}}{\partial t}+\frac{\mu}{h} \frac{\partial q}{\partial x}\right]_{b}(\delta \varphi)(t, b) \\
+ & \int_{0}^{\ell}\left[\left(-\frac{1}{2} \rho \frac{q^{2}}{h^{2}}-\rho g h-\rho \frac{\partial \lambda_{1}}{\partial t}\right)(\delta h)\right] \mathrm{d} x-\int_{a}^{b}\left[\lambda_{2}(\delta h)\right] \mathrm{d} x \\
& \left.+\left(\int_{a}^{b} \lambda_{2} \mathrm{~d} x-M \ddot{H}-M g\right)(\delta H)\right\} \mathrm{d} t .
\end{aligned}
$$

Since $\delta \mathcal{A}=0$ for any virtual displacement, we get the following system of equations

$$
\begin{array}{rlrl}
\frac{\partial}{\partial t}\left(\frac{q}{h}\right)-\frac{\partial}{\partial x} \frac{\partial \lambda_{1}}{\partial t} & =\frac{\mu}{\rho} \frac{\partial}{\partial x}\left(\frac{1}{h} \frac{\partial q}{\partial x}\right) & (t>0, x \in[0, \ell]), \\
{\left[\rho \frac{\partial \lambda_{1}}{\partial t}+\frac{\mu}{h} \frac{\partial q}{\partial x}\right]_{a}} & =0 & & (t>0), \\
{\left[\rho \frac{\partial \lambda_{1}}{\partial t}+\frac{\mu}{h} \frac{\partial q}{\partial x}\right]_{b}} & =0 & (t>0), \\
-\frac{\partial \lambda_{1}}{\partial t} & =\frac{1}{2} \frac{q^{2}}{h^{2}}+g h+\frac{\lambda_{2}}{\rho} & & (t>0, x \in \mathcal{I}), \\
-\frac{\partial \lambda_{1}}{\partial t} & =\frac{1}{2} \frac{q^{2}}{h^{2}}+g h & & (t>0, x \in \mathcal{E}) \\
M \ddot{H} & =\int_{a}^{b} \lambda_{2} \mathrm{~d} x-M g & (t>0) .
\end{array}
$$

We remark that equation (2.21) gives an interpretation to the Lagrange multiplier $\lambda_{2}$ as the pressure force exerted by the fluid on the solid. For this reason, we write in what follows

$$
p(t, x):=\lambda_{2}(t, x) .
$$

Combining equations (2.16)-(2.20), we can eliminate the Lagrange multiplier $\lambda_{1}$ and we obtain:

$$
\begin{array}{rlrl}
\frac{\partial h}{\partial t}+\frac{\partial q}{\partial x} & =0 & (t>0, x \in \mathcal{I} \cup \mathcal{E}), \\
\frac{\partial}{\partial t}\left(\frac{q}{H}\right)+\frac{\partial}{\partial x}\left(\frac{1}{2} \frac{q^{2}}{H^{2}}+g H+\frac{p}{\rho}\right) & =\frac{\mu}{\rho H} \frac{\partial^{2} q}{\partial x^{2}} & (t>0, x \in \mathcal{I}), \\
\frac{\partial}{\partial t}\left(\frac{q}{h}\right)+\frac{\partial}{\partial x}\left(\frac{1}{2} \frac{q^{2}}{h^{2}}+g h\right) & =\frac{\mu}{\rho} \frac{\partial}{\partial x}\left(\frac{1}{h} \frac{\partial q}{\partial x}\right) & (t>0, x \in \mathcal{E}), \\
{\left[\left(\frac{1}{2} \frac{q^{2}}{H^{2}}+g H+\frac{p}{\rho}\right)-\frac{\mu}{H \rho} \frac{\partial q}{\partial x}\right]\left(a^{+}\right)} & =\left[\left(\frac{1}{2} \frac{q^{2}}{h^{2}}+g h\right)-\frac{\mu}{h \rho} \frac{\partial q}{\partial x}\right]\left(a^{-}\right) \quad(t>0), \\
{\left[\left(\frac{1}{2} \frac{q^{2}}{H^{2}}+g H+\frac{p}{\rho}\right)-\frac{\mu}{H \rho} \frac{\partial q}{\partial x}\right]\left(b^{-}\right)} & =\left[\left(\frac{1}{2} \frac{q^{2}}{h^{2}}+g h\right)-\frac{\mu}{h \rho} \frac{\partial q}{\partial x}\right]\left(b^{+}\right) \quad(t>0), \\
M \ddot{H}(t) & =-M g+\int_{a}^{b} p(t, x) \mathrm{d} x \quad \quad(t>0),
\end{array}
$$

where we have also rewritten (2.2) and used (2.3) in the solid part. In the solid part we can simplify once again this system, using the fact that as $h(t, x)=H(t)$ does not depend on $x$, then equation (2.22) implies that $q$ is a first degree polynomial. 
Then our model for the rigid rectangle solid, constrained to move in the vertical direction, which is floating at the surface of a viscous fluid is

$$
\begin{aligned}
\frac{\partial h}{\partial t}+\frac{\partial q}{\partial x} & =0 & (t>0, x \in \mathcal{I} \cup \mathcal{E}), \\
\frac{\partial}{\partial t}\left(\frac{q}{H}\right)+\frac{\partial}{\partial x}\left(\frac{1}{2} \frac{q^{2}}{H^{2}}+g H+\frac{p}{\rho}\right) & =0 & (t>0, x \in \mathcal{I}), \\
\frac{\partial}{\partial t}\left(\frac{q}{h}\right)+\frac{\partial}{\partial x}\left(\frac{1}{2} \frac{q^{2}}{h^{2}}+g h\right) & =\frac{\mu}{\rho} \frac{\partial}{\partial x}\left(\frac{1}{h} \frac{\partial q}{\partial x}\right) & (t>0, x \in \mathcal{E}), \\
{\left[\left(\frac{1}{2} \frac{q^{2}}{H^{2}}+g H+\frac{p}{\rho}\right)-\frac{\mu}{H \rho} \frac{\partial q}{\partial x}\right]\left(t, a^{+}\right) } & =\left[\left(\frac{1}{2} \frac{q^{2}}{h^{2}}+g h\right)-\frac{\mu}{h \rho} \frac{\partial q}{\partial x}\right]\left(t, a^{-}\right) & (t>0), \\
{\left[\left(\frac{1}{2} \frac{q^{2}}{H^{2}}+g H+\frac{p}{\rho}\right)-\frac{\mu}{H \rho} \frac{\partial q}{\partial x}\right]\left(t, b^{-}\right) } & =\left[\left(\frac{1}{2} \frac{q^{2}}{h^{2}}+g h\right)-\frac{\mu}{h \rho} \frac{\partial q}{\partial x}\right]\left(t, b^{+}\right) & (t>0), \\
M \ddot{H}(t) & =-M g+\int_{a}^{b} p(t, x) \mathrm{d} x & (t>0) .
\end{aligned}
$$

Remark 2.1. As mentioned above, our modeling approach yields a nonstandard viscous term in the Saint-Venant system, more precisely in the right hand side of (2.31). Indeed, different forms of this term are generally used in most of the literature on viscous Saint-Venant equations. We mention in this direction Kloeden [13], Sundbye [19], Gerbeau and Perthame [9] or the review paper Bresch [5], which consider, once written in dimension one and with our notation, the term $\frac{\mu}{h \rho} \frac{\partial}{\partial x}\left(h \frac{\partial}{\partial x}\left(\frac{q}{h}\right)\right)$ in the right hand side of (2.31). However, other viscous terms have been considered in the literature, see, for instance, [5], Bernardi and Pironneau [3], Orenga [16] or Rodríguez and Taboada-Vázquez [18]. Comparing the viscosity term we introduce with the more commonly one used used, for instance, in [9], shows that their difference is given by

$$
\frac{\mu}{\rho} \frac{\partial}{\partial x}\left(\frac{1}{h} \frac{\partial q}{\partial x}\right)-\frac{\mu}{h \rho} \frac{\partial}{\partial x}\left(h \frac{\partial}{\partial x}\left(\frac{q}{h}\right)\right)=\frac{\mu q}{\rho h^{2}} \frac{\partial^{2} h}{\partial x^{2}}-\frac{\mu q}{\rho h^{3}}\left(\frac{\partial h}{\partial x}\right)^{2} .
$$

Passing to dimensionless variables and denoting by $\varepsilon$ the size of the solution, the term in the right hand side of the above formula is of higher order in $\varepsilon$ than the other terms in the momentum equation. Therefore, within the global precision of the shallow water approximation, the two viscosity terms are equivalent. Our choice of the viscous term in the right hand side of (2.31) seems an appropriate one in the case of viscous shallow water model of a fluid interacting with a floating solid for at least two reasons:

- It is energetically consistent, see Proposition 3.1 below.

- In our case, the smoothing effect coming from the viscous term applies to the flux $q$ instead of the velocity field $v$ as in [13] and [19] (note that in our case, unlike in the case of non viscous Saint Venant equations, $q$ and $v$ have different regularity properties). An advantage of our approach is that, since we use the variables $h$ and $q$ we do not need any information on the sign of the velocity $v$ at the solid-fluid interface. 


\section{Energy estimate and main result}

By extending the pressure by 0 in the fluid part, by taking $\rho=1$ and by adding boundary and initial conditions, we can rewrite system (2.28)-(2.33) as follows:

$$
\begin{array}{rlrl}
\frac{\partial h}{\partial t}+\frac{\partial q}{\partial x} & =0 & & (t>0, x \in \mathcal{I} \cup \mathcal{E}), \\
\frac{\partial}{\partial t}\left(\frac{q}{h}\right)+\frac{\partial}{\partial x}\left(\frac{q^{2}}{2 h^{2}}+g h+p\right)-\mu \frac{\partial}{\partial x}\left(\frac{1}{h} \frac{\partial q}{\partial x}\right) & =0 & (t>0, x \in \mathcal{I} \cup \mathcal{E}), \\
q(t, 0)=q(t, \ell) & =0 & (t>0), \\
p(t, x) & =0 & (t \geqslant 0, x \in \mathcal{E}), \\
h(t, x) & =H(t) & (t \geqslant 0, x \in \mathcal{I}), \\
{[q(t, \cdot)]_{a}=[q(t, \cdot)]_{b}} & =0 & (t>0), & \\
{\left[p(t, \cdot)+\frac{q^{2}(t, a)}{2 h^{2}(t, \cdot)}+g h(t, \cdot)-\frac{\mu}{h} \frac{\partial q}{\partial x}(t, \cdot)\right]_{a}} & =0 & (t \geqslant 0), & \\
{\left[p(t, \cdot)+\frac{q^{2}(t, b)}{2 h^{2}(t, \cdot)}+g h(t, \cdot)-\frac{\mu}{h} \frac{\partial q}{\partial x}(t, \cdot)\right]_{b}} & =0 & (t \geqslant 0), & \\
M \ddot{H}(t) & =-M g+\int_{a}^{b} p(t, x) \mathrm{d} x \quad(t>0), \\
h(0, x) & =h_{0}(x) & (x \in \mathcal{E}), \\
q(0, x) & =q_{0}(x) & (x \in[0, \ell]), \\
H(0) & =H_{0} . &
\end{array}
$$

Note that from (3.1) and (3.5) it follows that

$$
\frac{\partial q}{\partial x}(t, x)=-\dot{H}(t) \quad(t \geqslant 0, x \in \mathcal{I}) .
$$

In particular, the initial velocity of the solid is given by

$$
\dot{H}(0)=-\frac{\partial q_{0}}{\partial x} .
$$

Let us note that the spatial derivatives in the above equations and in the whole paper correspond to the spatial derivatives of the restrictions for each subdomain. In particular, we have the classical formula for any function $f$, smooth on $\mathcal{E} \cup \mathcal{I}$ :

$$
\int_{0}^{\ell} \frac{d f}{d x} d x=f(\ell)-f(0)-[f]_{b}-[f]_{a} .
$$

We first show that the system (3.1)-(3.12) satisfies an energy estimate:

Proposition 3.1. Let us write

$$
E(t)=\frac{1}{2} \int_{0}^{\ell}\left(\frac{q^{2}(t, x)}{h(t, x)}+g h^{2}(t, x)\right) \mathrm{d} x+\frac{1}{2} M \dot{H}^{2}(t)+M g H(t) \quad(t \geqslant 0)
$$

and let us consider a smooth solution $(h, q, H, p)$ of system (3.1)-(3.12). Then we have the following relation:

$$
\dot{E}(t)=-\mu \int_{0}^{\ell} \frac{1}{h(t, x)}\left[\frac{\partial q}{\partial x}(t, x)\right]^{2} \mathrm{~d} x \quad(t \geqslant 0) .
$$


Proof. From (3.15), it follows that

$$
\dot{E}(t)=\int_{0}^{\ell}\left[\frac{1}{2}\left(\frac{q}{h}\right)^{2} \frac{\partial h}{\partial t}+q \frac{\partial}{\partial t}\left(\frac{q}{h}\right)+g h \frac{\partial h}{\partial t}\right] \mathrm{d} x+M \dot{H}(t) \ddot{H}(t)+M g \dot{H}(t) .
$$

By combining the above formula with (3.1), (3.2) and (3.4) it follows that

$$
\begin{aligned}
\dot{E}(t)=-\int_{0}^{\ell}\left[\left(\frac{q^{2}}{2 h^{2}}\right.\right. & \left.+g h) \frac{\partial q}{\partial x}+q \frac{\partial}{\partial x}\left(\frac{q^{2}}{2 h^{2}}+g h\right)-q \mu \frac{\partial}{\partial x}\left(\frac{1}{h} \frac{\partial q}{\partial x}\right)\right] \mathrm{d} x \\
& -\int_{a}^{b} q(t, x) \frac{\partial p}{\partial x}(t, x) \mathrm{d} x+M \dot{H}(t) \ddot{H}(t)+M g \dot{H}(t) \quad(t \geqslant 0) .
\end{aligned}
$$

Since

$$
\left(\frac{q^{2}}{2 h^{2}}+g h\right) \frac{\partial q}{\partial x}+q \frac{\partial}{\partial x}\left(\frac{q^{2}}{2 h^{2}}+g h\right)=\frac{\partial}{\partial x}\left[q\left(\frac{q^{2}}{2 h^{2}}+g h\right)\right],
$$

we can combine (3.3), (3.6) and (3.17) to obtain

$$
\begin{aligned}
& \dot{E}(t)=q(t, a)\left[\frac{q^{2}(t, a)}{2 h^{2}(t, \cdot)}+g h(t, \cdot)\right]_{a}+q(t, b)\left[\frac{q^{2}(t, b)}{2 h^{2}(t, \cdot)}+g h(t, \cdot)\right]_{b} \\
& \quad+\int_{0}^{\ell}\left[q \mu \frac{\partial}{\partial x}\left(\frac{1}{h} \frac{\partial q}{\partial x}\right)\right] \mathrm{d} x-\int_{a}^{b} q(t, x) \frac{\partial p}{\partial x}(t, x) \mathrm{d} x+M \dot{H}(t) \ddot{H}(t)+M g \dot{H}(t) \quad(t \geqslant 0) .
\end{aligned}
$$

Integrating by parts in the two integrals in the above formula and using (3.7) and (3.8), together with (3.6), it follows that

$$
\dot{E}(t)=-\mu \int_{0}^{\ell} \frac{1}{h}\left(\frac{\partial q}{\partial x}\right)^{2} \mathrm{~d} x+\int_{a}^{b} p(t, x) \frac{\partial q}{\partial x}(t, x) \mathrm{d} x+M \dot{H}(t) \ddot{H}(t)+M g \dot{H}(t) \quad(t \geqslant 0) .
$$

Finally, inserting (3.13) and (3.9) in the last formula we obtain the desired energy estimate (3.16).

One can check that the stationary solutions of (3.1)-(3.9) can be parametrized by $\bar{H}>0$ by setting

$$
\bar{h}:=\bar{H}+\frac{M}{b-a}, \quad \bar{p}:=\frac{M g}{b-a}
$$

and in that case

$$
h^{S}(x)=\left\{\begin{array}{ll}
\bar{h} & x \in \mathcal{E} \\
\bar{H} & x \in \mathcal{I}
\end{array}, \quad q^{S}(x)=0 \quad \text { and } \quad p^{S}(x)=\left\{\begin{array}{ll}
0 & x \in \mathcal{E} \\
\bar{p} & x \in \mathcal{I}
\end{array} .\right.\right.
$$

In the statements below, we also need the following notation: we write $\mathcal{P}_{k}(\mathcal{I}), k \geqslant 0$ the set of polynomial functions of degree $\leqslant k$. Let us state our main results:

Theorem 3.2. Let us assume that

$$
\left[H_{0}, h_{0}, q_{0}\right]^{\top} \in \mathbb{R} \times H^{1}(\mathcal{E}) \times H^{1}(0, \ell)
$$

with

$$
\begin{gathered}
H_{0}>0, \quad h_{0}>0 \quad \text { in } \mathcal{E}, \quad H_{0}<\min \left(h_{0}\left(a^{-}\right), h_{0}\left(b^{+}\right)\right) \\
q_{0}(0)=q_{0}(\ell)=0 .
\end{gathered}
$$

Let $K>0$ be such that

$$
\left|H_{0}\right|+\left\|h_{0}\right\|_{H^{1}(\mathcal{E})}+\left\|q_{0}\right\|_{H^{1}(0, \ell)} \leqslant K, \quad \frac{1}{K} \leqslant h_{0}(x) \leqslant K \text { for } x \in \mathcal{E} .
$$


Then, there exists $T>0$, depending only on $K$ such that the system (3.1)-(3.12) admits a unique strong solution

$$
\begin{aligned}
H \in H^{2}(0, T), \quad h & \in H^{1}\left(0, T ; H^{1}(\mathcal{E})\right) \cap C^{1}\left([0, T] ; L^{2}(\mathcal{E})\right), \\
q & \in C^{0}\left([0, T] ; H^{1}(0, \ell)\right) \\
q_{\mid \mathcal{E}} \in H^{1}\left(0, T ; L^{2}(\mathcal{E})\right) & \cap C^{0}\left([0, T] ; H^{1}(\mathcal{E})\right) \cap L^{2}\left(0, T ; H^{2}(\mathcal{E})\right), \\
q_{\mid \mathcal{I}} & \in H^{1}\left(0, T ; \mathcal{P}_{1}(\mathcal{I})\right), \\
p_{\mid \mathcal{I}} & \in L^{2}\left(0, T ; \mathcal{P}_{2}(\mathcal{I})\right) .
\end{aligned}
$$

Moreover, there exists a constant $K_{T}>0$ such that

$$
\begin{gathered}
\frac{1}{K_{T}} \leqslant h_{0}(t, x), H(t) \leqslant K_{T}, \quad \text { for all } t \in(0, T), x \in \mathcal{E}, \\
H(t)<\min \left(h\left(t, a^{-}\right), h\left(t, b^{+}\right)\right) \quad \text { for all } t \in(0, T) .
\end{gathered}
$$

Theorem 3.3. For any $\bar{H}>0$, the system (3.1)-(3.12) is locally well-posed around the stationary state defined by (3.18)-(3.19). More precisely, there exists $\eta_{0}>0$ such that, for all $\eta \in\left(0, \eta_{0}\right)$ there exists two constants $\varepsilon>0$ and $C>0$, such that, for any

$$
\left[H_{0}, h_{0}, q_{0}\right]^{\top} \in \mathbb{R} \times H^{1}(\mathcal{E}) \times H^{1}(0, \ell)
$$

with

$$
\begin{gathered}
\int_{\mathcal{E}} h_{0}(x) \mathrm{d} x+H_{0}(b-a)=M \frac{|\mathcal{E}|}{|\mathcal{I}|}+\bar{H} \ell, \quad q_{0}(0)=q_{0}(\ell)=0, \\
\left|H_{0}-\bar{H}\right|+\left\|h_{0}-\bar{h}\right\|_{H^{1}(\mathcal{E})}+\left\|q_{0}\right\|_{H^{1}(0, \ell)} \leqslant \varepsilon
\end{gathered}
$$

there exists a unique solution of (3.1)-(3.12) with

$$
\begin{gathered}
H \in \bar{H}+H^{2}(0, \infty), \quad h \in \bar{h}+H^{1}\left(0, \infty ; H^{1}(\mathcal{E})\right) \cap C_{b}^{1}\left([0, \infty) ; L^{2}(\mathcal{E})\right), \\
q \in C_{b}\left([0, \infty) ; H^{1}(0, \ell)\right) \\
q_{\mid \mathcal{E}} \in H^{1}\left(0, \infty ; L^{2}(\mathcal{E})\right) \cap C_{b}\left([0, \infty) ; H^{1}(\mathcal{E})\right) \cap L^{2}\left(0, \infty ; H^{2}(\mathcal{E})\right), \\
q_{\mid \mathcal{I}} \in H^{1}\left(0, \infty ; \mathcal{P}_{1}(\mathcal{I})\right) \\
p_{\mid \mathcal{I}} \in \frac{M g}{|\mathcal{I}|}+L^{2}\left(0, \infty ; \mathcal{P}_{2}(\mathcal{I})\right) \\
\int_{\mathcal{E}} h(t, x) \mathrm{d} x+H(t)(b-a)=M \frac{|\mathcal{E}|}{|\mathcal{I}|}+\bar{H} \ell \quad(t \geqslant 0), \\
h(t, x) \geqslant \frac{\bar{h}}{2}, \quad H(t) \geqslant \frac{\bar{H}}{2}, \text { for all } t \in(0, \infty), x \in \mathcal{E} \\
H(t)<\min \left(h\left(t, a^{-}\right), h\left(t, b^{+}\right)\right) \quad \text { for all } t \in(0, \infty)
\end{gathered}
$$

satisfying the estimate

$$
\begin{aligned}
\left\|e^{\eta(\cdot)}(H-\bar{H})\right\|_{H^{2}(0, \infty)}+\left\|e^{\eta(\cdot)}(h-\bar{h})\right\|_{H^{1}\left(0, \infty ; H^{1}(\mathcal{E})\right)}+\left\|e^{\eta(\cdot)} q\right\|_{L^{\infty}\left(0, \infty ; H^{1}(0, \ell)\right)} \\
+\left\|\left.e^{\eta(\cdot)} q\right|_{\mathcal{E}}\right\|_{H^{1}\left(0, \infty ; L^{2}(\mathcal{E})\right) \cap L^{2}\left(0, \infty ; H^{2}(\mathcal{E})\right)} \leqslant C \varepsilon
\end{aligned}
$$

\section{Equations (3.1)-(3.12) as a parabolic system on $\mathcal{E}$}

In this section we eliminate pressure from (3.1)-(3.12) and obtain an equivalent system involving the restrictions of $h$ and $q$ to $\mathcal{E}$, together with the traces of $q$ at $x=a, b$ and $H$. More precisely, let us set

$$
q_{a}(t):=q\left(t, a^{-}\right)=q\left(t, a^{+}\right), \quad q_{b}(t):=q\left(t, b^{-}\right)=q\left(t, b^{+}\right) .
$$


From (3.13), we deduce

$$
\dot{H}=-\frac{q_{b}-q_{a}}{b-a} \quad \text { in } \mathcal{I}
$$

and

$$
q(t, x)=q_{a}(t)\left(\frac{x-b}{a-b}\right)+q_{b}(t)\left(\frac{x-a}{b-a}\right), \quad \frac{\partial q}{\partial x}(t, x)=\frac{q_{b}(t)-q_{a}(t)}{b-a} \quad(t \geqslant 0, x \in \mathcal{I}) .
$$

Combining (3.2), (3.5) and (3.13), we get

$$
\frac{\partial p}{\partial x}=-H \frac{\partial}{\partial t}\left(\frac{q}{H^{2}}\right)=-\frac{\dot{q}}{H}+2 \frac{q \dot{H}}{H^{2}} \quad(t \geqslant 0, x \in \mathcal{I}) .
$$

We recall that if $f \in \mathcal{P}_{2}([a, b])$, then we have the following standard formulas

$$
\begin{aligned}
& \int_{a}^{b} f(x) d x=f(a)(b-a)+f^{\prime}(a) \frac{(b-a)^{2}}{3}+f^{\prime}(b) \frac{(b-a)^{2}}{6}, \\
& \int_{a}^{b} f(x) d x=f(b)(b-a)-f^{\prime}(a) \frac{(b-a)^{2}}{6}-f^{\prime}(b) \frac{(b-a)^{2}}{3} .
\end{aligned}
$$

In particular, since $p$ is a second degree polynomial with respect to $x$, we deduce

$$
\begin{aligned}
& \int_{a}^{b} p(t, x) d x \\
& =p(t, a)(b-a)+\left(-\frac{\dot{q}_{a}(t)}{H(t)}+2 \frac{q_{a}(t) \dot{H}(t)}{H(t)^{2}}\right) \frac{(b-a)^{2}}{3}+\left(-\frac{\dot{q}_{b}(t)}{H(t)}+2 \frac{q_{b}(t) \dot{H}(t)}{H(t)^{2}}\right) \frac{(b-a)^{2}}{6}
\end{aligned}
$$

and

$$
\begin{aligned}
& \int_{a}^{b} p(t, x) d x \\
& =p(t, b)(b-a)-\left(-\frac{\dot{q}_{a}(t)}{H(t)}+2 \frac{q_{a}(t) \dot{H}(t)}{H(t)^{2}}\right) \frac{(b-a)^{2}}{6}-\left(-\frac{\dot{q}_{b}(t)}{H(t)}+2 \frac{q_{b}(t) \dot{H}(t)}{H^{2}}\right) \frac{(b-a)^{2}}{3} .
\end{aligned}
$$

Combining (4.7), (4.8), (4.2) and (3.9) yields

$$
\begin{aligned}
& {\left[M+\frac{(b-a)^{3}}{3 H}\right] \dot{q}_{a}-\left[M-\frac{(b-a)^{3}}{6 H}\right] \dot{q}_{b}=-M g }(b-a)+p(\cdot, a)(b-a)^{2} \\
&+\left[2 q_{a}^{2}-q_{a} q_{b}-q_{b}^{2}\right] \frac{(b-a)^{2}}{3 H^{2}} \quad(t>0),
\end{aligned}
$$

and

$$
\begin{aligned}
&-\left[M-\frac{(b-a)^{3}}{6 H}\right] \dot{q}_{a}+\left[M+\frac{(b-a)^{3}}{3 H}\right] \dot{q}_{b}= M g(b-a)-p(\cdot, b)(b-a)^{2} \\
&-\left[2 q_{b}^{2}-q_{a} q_{b}-q_{a}^{2}\right] \frac{(b-a)^{2}}{3 H^{2}} \quad(t>0) .
\end{aligned}
$$

Inverting the above linear system, we get

$$
\left[\begin{array}{c}
\dot{q}_{a} \\
\dot{q}_{b}
\end{array}\right]=S(H)\left[\begin{array}{c}
-M g(b-a)+p(\cdot, a)(b-a)^{2}+\left[2 q_{a}^{2}-q_{a} q_{b}-q_{b}^{2}\right] \frac{(b-a)^{2}}{3 H^{2}} \\
M g(b-a)-p(\cdot, b)(b-a)^{2}-\left[2 q_{b}^{2}-q_{a} q_{b}-q_{a}^{2}\right] \frac{(b-a)^{2}}{3 H^{2}}
\end{array}\right],
$$


where for all $H>0, S(H)$ is the following symmetric positive matrix:

$$
S(H):=\frac{H}{(b-a)^{3}\left(M+\frac{(b-a)^{3}}{12 H}\right)}\left[\begin{array}{ll}
M+\frac{(b-a)^{3}}{3 H} & M-\frac{(b-a)^{3}}{6 H} \\
M-\frac{(b-a)^{3}}{6 H} & M+\frac{(b-a)^{3}}{3 H}
\end{array}\right] .
$$

Considering equations (3.7)-(3.8) together with (4.3) and with (4.11), we deduce, we obtain:

$$
\left[\begin{array}{c}
\dot{q}_{a} \\
\dot{q}_{b}
\end{array}\right]=R\left(H, q(\cdot, a), \frac{\partial}{\partial x} q\left(\cdot, a^{-}\right), h\left(\cdot, a^{-}\right), q(\cdot, b), \frac{\partial}{\partial x} q\left(\cdot, b^{+}\right), h\left(\cdot, b^{+}\right)\right),
$$

where

$$
\begin{aligned}
R\left(H, q(\cdot, a), \frac{\partial}{\partial x} q\left(\cdot, a^{-}\right), h\left(\cdot, a^{-}\right), q(\cdot, b), \frac{\partial}{\partial x} q\left(\cdot, b^{+}\right), h\left(\cdot, b^{+}\right)\right) \\
=S(H)\left[\begin{array}{l}
-M g(b-a)+p(\cdot, a)(b-a)^{2}+\left[2 q_{a}^{2}-q_{a} q_{b}-q_{b}^{2}\right] \frac{(b-a)^{2}}{3 H^{2}} \\
M g(b-a)-p(\cdot, b)(b-a)^{2}-\left[2 q_{b}^{2}-q_{a} q_{b}-q_{a}^{2}\right] \frac{(b-a)^{2}}{3 H^{2}}
\end{array}\right],
\end{aligned}
$$

and $p(\cdot, a)$ and $p(\cdot, b)$ are given by:

$$
\begin{aligned}
& p(t, a)=\frac{q^{2}(t, a)}{2 h^{2}\left(t, a^{-}\right)}+g h\left(t, a^{-}\right)-\frac{\mu}{h} \frac{\partial q}{\partial x}\left(t, a^{-}\right)-\frac{q^{2}(t, a)}{2 H^{2}(t)}-g H(t)+\mu \frac{q_{b}(t)-q_{a}(t)}{H(b-a)} \\
& p(t, b)=\frac{q^{2}(t, b)}{2 h^{2}\left(t, b^{+}\right)}+g h\left(t, b^{+}\right)-\frac{\mu}{h} \frac{\partial q}{\partial x}\left(t, b^{+}\right)-\frac{q^{2}(t, b)}{2 H^{2}(t)}-g H(t)+\mu \frac{q_{b}(t)-q_{a}(t)}{H(b-a)} .
\end{aligned}
$$

Finally, the system (3.1)-(3.12) writes in the equivalent form

$$
\begin{gathered}
\dot{H}=-\frac{q_{b}-q_{a}}{b-a} \quad(t \geqslant 0), \\
\frac{\partial h}{\partial t}(t, x)+\frac{\partial q}{\partial x}(t, x)=0 \quad(t>0, x \in \mathcal{E}), \\
\frac{\partial q}{\partial t}+\frac{\partial}{\partial x}\left(\frac{q^{2}}{h}+\frac{g h^{2}}{2}\right)=h \frac{\partial}{\partial x}\left(\frac{\mu}{h} \frac{\partial q}{\partial x}\right) \\
q(t, 0)=q(t, \ell)=0 \quad(t>0, x \in \mathcal{E}), \\
q(t, a)=q_{a}(t), \quad q(t, b)=q_{b}(t) \\
{\left[\begin{array}{c}
\dot{q}_{a} \\
\dot{q}_{b}
\end{array}\right]=R\left(H, q(\cdot, a), \frac{\partial}{\partial x} q\left(\cdot, a^{-}\right), h\left(\cdot, a^{-}\right), q(\cdot, b), \frac{\partial}{\partial x} q\left(\cdot, b^{+}\right), h\left(\cdot, b^{+}\right)\right),}
\end{gathered}
$$

with $R$ defined by (4.14), (4.15) and (4.16).

\section{$5 \quad$ Local in time existence and uniqueness}

In this section we prove Theorem 3.2. In view of the reduction performed in Section 4, our main result in Theorem 3.2 can be restated as: 
Theorem 5.1. Assume that

$$
\left[H_{0}, h_{0}, q_{0}, q_{a, 0}, q_{b, 0}\right]^{\top} \in \mathbb{R} \times H^{1}(\mathcal{E}) \times H^{1}(\mathcal{E}) \times \mathbb{R}^{2},
$$

with

$$
\begin{gathered}
H_{0}>0, \quad h_{0}>0 \quad \text { in } \mathcal{E}, \quad H_{0}<\min \left(h_{0}\left(a^{-}\right), h_{0}\left(b^{+}\right)\right), \\
q_{0}(0)=q_{0}(\ell)=0, \quad q_{0}(a)=q_{a, 0}, \quad q_{0}(b)=q_{b, 0} .
\end{gathered}
$$

Let $K>0$ be such that

$$
\left|H_{0}\right|+\left\|h_{0}\right\|_{H^{1}(\mathcal{E})}+\left\|q_{0}\right\|_{H^{1}(\mathcal{E})}+\left|q_{0, a}\right|+\left|q_{0, b}\right| \leqslant K, \quad \frac{1}{K} \leqslant h_{0}(x) \leqslant K \text { for } x \in \mathcal{E} .
$$

Then, there exists $T>0$, depending only on $K$, such that the system (4.17)-(4.22) admits a unique strong solution

$$
\begin{gathered}
H \in H^{2}(0, T), \quad h \in H^{1}\left(0, T ; H^{1}(\mathcal{E})\right) \cap C^{1}\left([0, T) ; L^{2}(\mathcal{E})\right), \\
q \in H^{1}\left(0, T ; L^{2}(\mathcal{E})\right) \cap C\left([0, T) ; H^{1}(\mathcal{E})\right) \cap L^{2}\left(0, T ; H^{2}(\mathcal{E})\right), \\
q_{a} \in H^{1}(0, T), \quad q_{b} \in H^{1}(0, T)
\end{gathered}
$$

Moreover, there exists a constant $K_{T}>0$ such that

$$
\begin{gathered}
\frac{1}{K_{T}} \leqslant h(t, x), H(t) \leqslant K_{T} \quad(t \in(0, T), x \in \mathcal{E}), \\
H(t)<\min \left(h\left(t, a^{-}\right), h\left(t, b^{+}\right)\right) \quad(t \in(0, T)) .
\end{gathered}
$$

The proof of the above theorem relies on classical estimates on linear parabolic problems, combined with the use of the Banach fixed point theorem. The strategy we adopt here is based on the fact that the system (4.17)-(4.22) can be rewritten as

$$
\begin{array}{ll}
\dot{H}=-\frac{q_{b}-q_{a}}{b-a} & (t>0), \\
\frac{\partial h}{\partial t}+\frac{\partial q}{\partial x}=0 & (t>0, x \in \mathcal{E}), \\
\frac{\partial q}{\partial t}-\mu \frac{\partial^{2} q}{\partial x^{2}}=\mathcal{F}_{1}(h, q) & (t>0, x \in \mathcal{E}), \\
q(t, 0)=q(t, \ell)=0, & (t>0), \\
q(t, a)=q_{a}(t), \quad q(t, b)=q_{b}(t) & (t>0), \\
{\left[\begin{array}{l}
\dot{q}_{a} \\
\dot{q}_{b}
\end{array}\right]=\mathcal{F}_{2}\left(H, q, q_{a}, q_{b}\right)} & (t>0), \\
h(0, x)=h_{0}(x), \quad q(0, x)=q_{0}(x) & (x \in \mathcal{E}), \\
H(0)=H_{0}, \quad q_{a}(0)=q_{a, 0}, \quad q_{b}(0)=q_{b, 0},
\end{array}
$$

where

$$
\mathcal{F}_{1}(h, q)=-\frac{2 q}{h} \frac{\partial q}{\partial x}+\frac{q^{2}}{h^{2}} \frac{\partial h}{\partial x}+g h \frac{\partial h}{\partial x}-\frac{\mu}{h} \frac{\partial h}{\partial x} \frac{\partial q}{\partial x},
$$

and

$$
\mathcal{F}_{2}\left(H, q, q_{a}, q_{b}\right)=R\left(H, q(\cdot, a), \frac{\partial}{\partial x} q\left(\cdot, a^{-}\right), h\left(\cdot, a^{-}\right), q(\cdot, b), \frac{\partial}{\partial x} q\left(\cdot, b^{+}\right), h\left(\cdot, b^{+}\right)\right),
$$

with $R$ defined by (4.14), (4.15) and (4.16). Next, by replacing $\mathcal{F}_{1}$ and $\mathcal{F}_{2}$ by given source term $f_{1}$ and $f_{2}$ we obtain the following linear system : 


$$
\begin{array}{ll}
\dot{H}=-\frac{q_{b}-q_{a}}{b-a} & (t>0), \\
\frac{\partial h}{\partial t}+\frac{\partial q}{\partial x}=0 & (t>0, x \in \mathcal{E}), \\
\frac{\partial q}{\partial t}-\mu \frac{\partial^{2} q}{\partial x^{2}}=f_{1} & (t>0, x \in \mathcal{E}), \\
q(t, 0)=q(t, \ell)=0, \quad(t>0), \\
q(t, a)=q_{a}(t), \quad q(t, b)=q_{b}(t) & (t>0), \\
{\left[\begin{array}{l}
\dot{q}_{a} \\
\dot{q}_{b}
\end{array}\right]=f_{2}} & (t>0), \\
h(0, x)=h_{0}(x), \quad q(0, x)=q_{0}(x) & (x \in \mathcal{E}), \\
H(0)=H_{0}, \quad q_{a}(0)=q_{a, 0}, \quad q_{b}(0)=q_{b, 0} .
\end{array}
$$

We have the following regularity result for the system (5.17)-(5.24)

Theorem 5.2. Let us assume that $\left[H_{0}, h_{0}, q_{0}, q_{a, 0}, q_{b, 0}\right]^{\top} \in \mathbb{R} \times H^{1}(\mathcal{E}) \times H^{1}(\mathcal{E}) \times \mathbb{R}^{2}$ and satisfies the compatibility condition (5.2). Then, for all $0<T \leqslant 1, f_{1} \in L^{2}\left(0, T ; L^{2}(\mathcal{E})\right)$ and $f_{2} \in L^{2}\left(0, T ; \mathbb{R}^{2}\right)$ the system (5.17)-(5.24) admits a unique solution

$$
\begin{gathered}
H \in H^{2}(0, T), \quad h \in H^{1}\left(0, T ; H^{1}(\mathcal{E})\right) \cap C^{1}\left([0, T) ; L^{2}(\mathcal{E})\right), \\
q \in H^{1}\left(0, T ; L^{2}(\mathcal{E})\right) \cap C\left([0, T) ; H^{1}(\mathcal{E})\right) \cap L^{2}\left(0, T ; H^{2}(\mathcal{E})\right), \\
q_{a} \in H^{1}(0, T), \quad q_{b} \in H^{1}(0, T) .
\end{gathered}
$$

Moreover, there exists a constant $C$ independent of $T$ such that

$$
\begin{array}{r}
\|H\|_{H^{2}(0, T)}+\|h\|_{H^{1}\left(0, T ; H^{1}(\mathcal{E})\right)}+\|h\|_{L^{\infty}\left(0, T ; H^{1}(\mathcal{E})\right)}+\|q\|_{L^{2}\left(0, T ; H^{2}(\mathcal{E})\right)}+\|q\|_{H^{1}\left(0, T ; L^{2}(\mathcal{E})\right)} \\
+\|q\|_{L^{\infty}\left(0, T ; H^{1}(\mathcal{E})\right)}+\left\|\left(q_{a}, q_{b}\right)\right\|_{H^{1}\left(0, T ; \mathbb{R}^{2}\right)} \\
+C\left(\left|H_{0}\right|+\left\|\widetilde{h}_{0}\right\|_{H^{1}(\mathcal{E})}+\left\|q_{0}\right\|_{H^{1}(\mathcal{E})}\right. \\
\left.+\left|q_{a, 0}\right|+\left|q_{b, 0}\right|+\left\|\left(f_{1}, f_{2}\right)\right\|_{L^{2}\left(0, T ; L^{2}(\mathcal{E}) \times \mathbb{R}^{2}\right)}\right),
\end{array}
$$

for all $0<T \leqslant 1$.

Proof. Let us remark that the linear system (5.17)-(5.24) can be solved in "cascades": Eq. (5.22) can be solved independently and admits a unique solution $\left(q_{a}, q_{b}\right) \in H^{1}\left(0, T ; \mathbb{R}^{2}\right)$. Next we solve Eq. (5.17) and we obtain $H \in H^{2}(0, T)$. Using the standard regularity results for parabolic equations with non-homogeneous boundary conditions, we also have that

$$
q \in L^{2}\left(0, T ; H^{2}(\mathcal{E})\right) \cap H^{1}\left(0, T ; L^{2}(\mathcal{E})\right) \cap L^{\infty}\left(0, T ; H^{1}(\mathcal{E})\right) .
$$

Finally, using the regularity of $q$, we obtain the desired regularity of $h$ from Eq. (5.18). To obtain estimates with continuity constant independent of time $T$ we can proceed as in $[10$, Theorem 5.3].

Now we estimate the nonlinear terms defined in (5.15)-(5.16).

Proposition 5.3. Let us assume that $\left[H_{0}, h_{0}, q_{0}, q_{a, 0}, q_{b, 0}\right]^{\top} \in \mathbb{R} \times H^{1}(\mathcal{E}) \times H^{1}(\mathcal{E}) \times \mathbb{R}^{2}$ such that (5.1)-(5.3) holds. There exist $\widetilde{T} \in(0,1), \delta>0$ and $C=C(M, \widetilde{T})>0$ such that for $T \in(0, \widetilde{T}]$ and for $\left(f_{1}, f_{2}\right) \in L^{2}\left(0, T ; L^{2}(\mathcal{E}) \times \mathbb{R}^{2}\right)$ satisfying

$$
\left\|\left(f_{1}, f_{2}\right)\right\|_{L^{2}\left(0, T ; L^{2}(\mathcal{E}) \times \mathbb{R}^{2}\right)} \leqslant 1,
$$

the solution $\left(H, h, q, q_{a}, q_{b}\right)$ of (5.17)-(5.24) verifies

$$
\left\|\mathcal{F}_{1}\right\|_{L^{2}\left(0, T ; L^{2}(\mathcal{E})\right)}+\left\|\mathcal{F}_{2}\right\|_{L^{2}\left(0, T ; \mathbb{R}^{2}\right)} \leqslant C T^{\delta} .
$$


Proof. We choose $\widetilde{T}<1$ and $T \in(0, \widetilde{T}]$. The constant appearing in this proof depends only on $K$ and independent of $T$. First of all, from (5.25) we first obtain

$$
\begin{array}{r}
\|H\|_{H^{2}(0, T)}+\|h\|_{H^{1}\left(0, T ; H^{1}(\mathcal{E})\right)}+\|h\|_{L^{\infty}\left(0, T ; H^{1}(\mathcal{E})\right)}+\|q\|_{L^{2}\left(0, T ; H^{2}(\mathcal{E})\right)}+\|q\|_{H^{1}\left(0, T ; L^{2}(\mathcal{E})\right)} \\
+\|q\|_{L^{\infty}\left(0, T ; H^{1}(\mathcal{E})\right)}+\left\|\left(q_{a}, q_{b}\right)\right\|_{H^{1}\left(0, T ; \mathbb{R}^{2}\right)} \leqslant C .
\end{array}
$$

Applying Hölder's inequality together with the above estimate we deduce that

$$
\left\|H-H_{0}\right\|_{L^{\infty}(0, T)} \leqslant C \sqrt{T}, \quad\left\|h-h_{0}\right\|_{L^{\infty}\left(0, T ; H^{1}(\mathcal{E})\right)} \leqslant C \sqrt{T} .
$$

In particular, there exists $\widetilde{T}$ such that for all $T \in(0, \widetilde{T}]$ the following holds:

$$
\begin{gathered}
H(t) \geqslant \frac{H_{0}}{2}, \quad h(t, x) \geqslant \frac{1}{2 K}, \quad t \in(0, T), x \in \mathcal{E}, \\
\|h\|_{L^{\infty}((0, T) \times \mathcal{E})}+\|H\|_{L^{\infty}(0, T)} \leqslant C, \\
\|h\|_{L^{2}\left(0, T ; H^{1}(\mathcal{E})\right)}+\quad\|H\|_{L^{2}(0, T)} \leqslant C \sqrt{T}, \\
\left\|\frac{1}{h}\right\|_{L^{\infty}\left(0, T ; H^{1}(\mathcal{E})\right)}+\left\|\frac{1}{h}\right\|_{L^{\infty}((0, T) \times \mathcal{E})}+\left\|\frac{1}{H}\right\|_{L^{\infty}(0, T)} \leqslant C .
\end{gathered}
$$

In a similar manner, we also obtain

$$
\left\|q_{a}\right\|_{L^{\infty}(0, T)}+\left\|q_{b}\right\|_{L^{\infty}(0, T)} \leqslant C, \quad\left\|q_{a}\right\|_{L^{2}(0, T)}+\left\|q_{b}\right\|_{L^{2}(0, T)} \leqslant C \sqrt{T} .
$$

On the other hand, using an interpolation inequality one has following estimate (see, for instance estimate, (6.13) of [10])

$$
\|q\|_{L^{2}\left(0, T ; H^{1+s}(\mathcal{E})\right)} \leqslant C T^{(1-s) / 4}, \quad s \in(0,1) .
$$

Using the above estimate, (5.26) and the Sobolev embedding we get

$$
\|q\|_{L^{\infty}((0, T) \times \mathcal{E})} \leqslant C, \quad\left\|\frac{\partial q}{\partial x}\right\|_{L^{2}\left(0, T ; L^{\infty}(\mathcal{E})\right)} \leqslant C T^{(1-s) / 4}, \quad s \in(1 / 2,1)
$$

We are now in a position to estimate the non-linear terms defined in (5.15)-(5.16).

Estimate of $\mathcal{F}_{1}$ :

$$
\left\|\mathcal{F}_{1}\right\|_{L^{2}\left(0, T ; L^{2}(\mathcal{E})\right)} \leqslant C T^{\delta}, \text { for some } \delta>0 \text {. }
$$

-Estimate of the first term of $\mathcal{F}_{1}$ : Using (5.26), (5.27) and (5.33), we have

$$
\left\|-\frac{2 q}{h} \frac{\partial q}{\partial x}\right\|_{L^{2}\left(0, T ; L^{2}(\mathcal{E})\right)} \leqslant C\|q\|_{L^{2}\left(0, T ; H^{1}(\mathcal{E})\right)} \leqslant C \sqrt{T}\|q\|_{L^{\infty}\left(0, T ; H^{1}(\mathcal{E})\right)} \leqslant C \sqrt{T} .
$$

-Estimate of the second and third term of $\mathcal{F}_{1}$ : Using (5.26), (5.27) and (5.33) we have

$$
\left\|\frac{q^{2}}{h^{2}} \frac{\partial h}{\partial x}+g h \frac{\partial h}{\partial x}\right\|_{L^{2}\left(0, T ; L^{2}(\mathcal{E})\right)} \leqslant C\|h\|_{L^{2}\left(0, T ; H^{1}(\mathcal{E})\right)} \leqslant C \sqrt{T} .
$$

-Estimate of the last term of $\mathcal{F}_{1}$ : Using (5.26), (5.27) and (5.33) we obtain

$$
\left\|\frac{\mu}{h} \frac{\partial h}{\partial x} \frac{\partial q}{\partial x},\right\|_{L^{2}\left(0, T ; L^{2}(\mathcal{E})\right)} \leqslant C\left\|\frac{\partial h}{\partial x}\right\|_{L^{\infty}\left(0, T ; L^{2}(\mathcal{E})\right)}\left\|\frac{\partial q}{\partial x}\right\|_{L^{2}\left(0, T ; L^{\infty}(\mathcal{E})\right)} \leqslant C T^{(1-s) / 4}
$$

for some $s \in(1 / 2,1)$. Thus we have proved (5.34).

Estimate of $\mathcal{F}_{2}$ :

$$
\left\|\mathcal{F}_{2}\right\|_{L^{2}\left(0, T ; \mathbb{R}^{2}\right)} \leqslant C T^{\delta}, \text { for some } \delta>0 .
$$


-Estimate of $S(H)$ defined in (4.12) : Using (5.27) - (5.29), we have the following estimates

$$
\|S(H)\|_{L^{\infty}\left(0, T ; \mathbb{R}^{4}\right)} \leqslant C, \quad\|S(H)\|_{L^{2}\left(0, T ; \mathbb{R}^{4}\right)} \leqslant C \sqrt{T} .
$$

-Estimate $p(t, a)$ and $p(t, b)$ defined in (4.15) - (4.16): We claim that

$$
\|p(\cdot, a)\|_{L^{2}(0, T)}+\|p(\cdot, b)\|_{L^{2}(0, T)} \leqslant C T^{\delta}, \text { for some } \delta>0 .
$$

We provide only the estimate of third term of $p(t, a)$. Estimates of the other terms are similar. Using (5.30) and (5.32) we have

$$
\left\|\frac{\mu}{h} \frac{\partial q}{\partial x}\left(\cdot, a^{-}\right)\right\|_{L^{2}(0, T)} \leqslant C\left\|\frac{1}{h} \frac{\partial q}{\partial x}\right\|_{L^{2}\left(0, T ; H^{s}(0, a)\right)} \leqslant C\left\|\frac{1}{h}\right\|_{L^{\infty}\left(0, T ; H^{1}(0, a)\right)}\left\|\frac{\partial q}{\partial x}\right\|_{L^{2}\left(0, T ; H^{s}(0, a)\right)} \leqslant C T^{(1-s) / 4},
$$

for some $s \in(1 / 2,1)$. Thus we have (5.37). Finally, combining (5.36), (5.37) and (5.31), we obtain $(5.35)$.

We are now in a position to prove one of Theorem 5.1.

Proof of Theorem 5.1. Let $\widetilde{T}$ be the constant in Proposition 5.3. For $T \in(0, \widetilde{T}]$, we consider the ball

$$
\mathfrak{B}_{T}:=\left\{\left(f_{1}, f_{2}\right) \in L^{2}\left(0, T ; L^{2}(\mathcal{E}) \times \mathbb{R}^{2}\right) ;\left\|\left(f_{1}, f_{2}\right)\right\|_{L^{2}\left(0, T ; L^{2}(\mathcal{E}) \times \mathbb{R}^{2}\right)} \leqslant 1\right\}
$$

and the map

$$
\Xi:\left(f_{1}, f_{2}\right) \in \mathfrak{B}_{T} \mapsto\left(\mathcal{F}_{1}(h, q), \mathcal{F}_{2}\left(H, q, q_{a}, q_{b}\right)\right),
$$

where $\left(H, h, q, q_{a}, q_{b}\right)$ is the solution to the system (5.17)-(5.24) and where $\mathcal{F}_{1}$ and $\mathcal{F}_{2}$ are given by (5.15)-(5.16). By Proposition 5.3, we have that $\Xi\left(\mathfrak{B}_{T}\right) \subset \mathfrak{B}_{T}$ for $T$ small enough. With similar calculation as Proposition 5.3, we can also show that for small $T, \Xi_{\mid \mathfrak{B}_{T}}$ is a strict contraction. This completes the proof of Theorem 5.1.

\section{Global in time existence and uniqueness}

In this section, we linearize the system (4.17)-(4.22) around a stationary state and we study the corresponding linear system. Note that, in order to prove global existence for small data of our original problem (4.17)-(4.22) we need this linearization to be exponentially stable, so that we cannot use the linearized problem (5.17)-(5.24), introduced for the local existence result.

\subsection{Linearization around a stationary state}

We consider the stationary state given by (3.19) and we define

$$
\widetilde{H}:=H-\bar{H}, \quad \widetilde{h}:=h-h^{S}=\left\{\begin{array}{ll}
h-\bar{h} & \text { in } \mathcal{E} \\
\widetilde{H} & \text { in } \mathcal{I}
\end{array} .\right.
$$

From (4.15)-(4.16) and (3.18), we deduce

$$
\begin{aligned}
& p(t, a)=\frac{q^{2}(t, a)}{2}\left(\frac{1}{(\widetilde{h}+\bar{h})^{2}\left(t, a^{-}\right)}-\frac{1}{(\widetilde{H}+\bar{H})^{2}(t)}\right)+g\left(\widetilde{h}\left(t, a^{-}\right)-\widetilde{H}(t)\right)+g \frac{M}{b-a} \\
& -\frac{\mu}{\bar{h}} \frac{\partial q}{\partial x}\left(t, a^{-}\right)+\mu \frac{q_{b}(t)-q_{a}(t)}{\bar{H}(b-a)}+\frac{\mu}{\bar{h}}\left(\frac{\widetilde{h}}{(\widetilde{h}+\bar{h})} \frac{\partial q}{\partial x}\right)\left(t, a^{-}\right)-\mu \frac{q_{b}(t)-q_{a}(t)}{b-a} \frac{\widetilde{H}}{(\widetilde{H}+\bar{H}) \bar{H}}
\end{aligned}
$$




$$
\begin{gathered}
p(t, b)=\frac{q^{2}(t, b)}{2}\left(\frac{1}{(\widetilde{h}+\bar{h})^{2}\left(t, b^{+}\right)}-\frac{1}{(\widetilde{H}+\bar{H})^{2}(t)}\right)+g\left(\widetilde{h}\left(t, b^{+}\right)-\widetilde{H}(t)\right)+g \frac{M}{b-a} \\
-\frac{\mu}{\bar{h}} \frac{\partial q}{\partial x}\left(t, b^{+}\right)+\mu \frac{q_{b}(t)-q_{a}(t)}{\bar{H}(b-a)}+\frac{\mu}{\bar{h}}\left(\frac{\widetilde{h}}{(\widetilde{h}+\bar{h})} \frac{\partial q}{\partial x}\right)\left(t, b^{+}\right)-\mu \frac{q_{b}(t)-q_{a}(t)}{b-a} \frac{\widetilde{H}}{(\widetilde{H}+\bar{H}) \bar{H}} .
\end{gathered}
$$

Thus

$$
\begin{aligned}
-M g(b-a)+p(\cdot, a)(b-a)^{2}+\left[2 q_{a}^{2}-q_{a} q_{b}-q_{b}^{2}\right] \frac{(b-a)^{2}}{3 H^{2}} & \\
=(b-a)^{2}\left\{g\left(\widetilde{h}\left(\cdot, a^{-}\right)-\widetilde{H}\right)-\frac{\mu}{\bar{h}} \frac{\partial q}{\partial x}\left(\cdot, a^{-}\right)+\frac{\mu\left(q_{b}-q_{a}\right)}{\bar{H}(b-a)}\right. & \quad \frac{q^{2}(\cdot, a)}{2}\left(\frac{1}{(\widetilde{h}+\bar{h})^{2}\left(\cdot, a^{-}\right)}-\frac{1}{(\widetilde{H}+\bar{H})^{2}}\right) \\
& \left.+\frac{\mu}{\bar{h}}\left(\frac{\widetilde{h}}{(\widetilde{h}+\bar{h})} \frac{\partial q}{\partial x}\right)\left(t, a^{-}\right)-\mu \frac{q_{b}-q_{a}}{b-a} \frac{\widetilde{H}}{(\widetilde{H}+\bar{H}) \bar{H}}+\frac{2 q_{a}^{2}-q_{a} q_{b}-q_{b}^{2}}{3(\widetilde{H}+\bar{H})^{2}}\right\}
\end{aligned}
$$

and

$$
\begin{aligned}
& M g(b-a)-p(\cdot, b)(b-a)^{2}- {\left[2 q_{a}^{2}-q_{a} q_{b}-q_{b}^{2}\right] \frac{(b-a)^{2}}{3 H^{2}} } \\
&=(b-a)^{2}\left\{-g\left(\widetilde{h}\left(\cdot, b^{+}\right)-\widetilde{H}\right)+\frac{\mu}{\bar{h}} \frac{\partial q}{\partial x}\left(\cdot, a^{-}\right)-\frac{\mu\left(q_{b}-q_{a}\right)}{\bar{H}(b-a)}\right. \quad-\frac{q^{2}(\cdot, b)}{2}\left(\frac{1}{(\widetilde{h}+\bar{h})^{2}\left(\cdot, b^{+}\right)}-\frac{1}{(\widetilde{H}+\bar{H})^{2}}\right) \\
&\left.+\frac{\mu}{\bar{h}}\left(\frac{\widetilde{h}}{(\widetilde{h}+\bar{h})} \frac{\partial q}{\partial x}\right)\left(t, b^{+}\right)+\mu \frac{q_{b}-q_{a}}{b-a} \frac{\widetilde{H}}{(\widetilde{H}+\bar{H}) \bar{H}}-\frac{2 q_{b}^{2}-q_{a} q_{b}-q_{a}^{2}}{3(\widetilde{H}+\bar{H})^{2}}\right\}
\end{aligned}
$$

Then $\left(\widetilde{H}, \widetilde{h}, q, q_{a}, q_{b}\right)$ satisfies

$$
\begin{array}{ccc}
\dot{\widetilde{H}}=-\frac{q_{b}-q_{a}}{b-a} & (t \geqslant 0), \\
\frac{\partial \widetilde{h}}{\partial t}(t, x)+\frac{\partial q}{\partial x}(t, x)=0 & (t>0, x \in \mathcal{E}), \\
\frac{\partial q}{\partial t}+g \bar{h} \frac{\partial \widetilde{h}}{\partial x}-\mu \frac{\partial^{2} q}{\partial x^{2}}=F_{1}(\widetilde{h}, q) & (t>0, x \in \mathcal{E}), \\
q(t, 0)=q(t, \ell)=0 & (t>0), \\
q(t, a)=q_{a}(t), \quad q(t, b)=q_{b}(t) & (t>0), \\
{\left[\begin{array}{l}
\dot{q}_{a} \\
\dot{q}_{b}
\end{array}\right]=(b-a)^{2} S(\bar{H})\left[\begin{array}{l}
g\left(\widetilde{h}\left(\cdot, a^{-}\right)-\widetilde{H}\right)-\frac{\mu}{\bar{h}} \frac{\partial q}{\partial x}\left(\cdot, a^{-}\right)+\frac{\mu}{\bar{H}} \frac{q(\cdot, b)-q(\cdot, a)}{b-a} \\
\left.-g\left(\widetilde{h}\left(\cdot, b^{+}\right)-\widetilde{H}\right)+\frac{\mu}{\bar{h}} \frac{\partial q}{\partial x}\left(\cdot, b^{+}\right)-\frac{\mu}{\bar{H}} \frac{q(\cdot, b)-q(\cdot, a)}{b-a}\right]
\end{array}\right]}
\end{array}
$$

where

$$
F_{1}(\widetilde{h}, q):=-\frac{\partial}{\partial x}\left(\frac{q^{2}}{\widetilde{h}+\bar{h}}+g \frac{\widetilde{h}^{2}}{2}\right)-\frac{\mu}{(\bar{h}+\widetilde{h})^{2}} \frac{\partial \widetilde{h}}{\partial x} \frac{\partial q}{\partial x}
$$


$F_{2}\left(\widetilde{H}, \widetilde{h}, q, q_{b}, q_{a}\right)$

$$
\begin{array}{r}
:=(b-a)^{2}(S(\widetilde{H}+\bar{H})-S(\bar{H}))\left[\begin{array}{r}
g\left(\widetilde{h}\left(\cdot, a^{-}\right)-\widetilde{H}\right)-\frac{\mu}{\bar{h}} \frac{\partial q}{\partial x}\left(\cdot, a^{-}\right)+\frac{\mu}{\bar{H}} \frac{q_{b}-q_{a}}{b-a} \\
\left.-g\left(\widetilde{h}\left(\cdot, b^{+}\right)-\widetilde{H}\right)+\frac{\mu}{\bar{h}} \frac{\partial q}{\partial x}\left(\cdot, a^{-}\right)-\frac{\mu}{\bar{H}} \frac{q_{b}-q_{a}}{b-a}\right] \\
+(b-a)^{2} S(\widetilde{H}+\bar{H})\left[\begin{array}{c}
F_{3}\left(\widetilde{H}, \widetilde{h}, q, q_{b}, q_{a}\right) \\
F_{4}\left(\widetilde{H}, \widetilde{h}, q, q_{b}, q_{a}\right)
\end{array}\right], \\
F_{3}\left(\widetilde{H}, \widetilde{h}, q, q_{b}, q_{a}\right):=\frac{q_{a}^{2}}{2}\left(\frac{1}{(\widetilde{h}+\bar{h})^{2}\left(\cdot, a^{-}\right)}-\frac{1}{(\widetilde{H}+\bar{H})^{2}}\right)+\frac{\mu}{\bar{h}\left(\frac{\widetilde{h}}{(\widetilde{h}+\bar{h})} \frac{\partial q}{\partial x}\right)\left(\cdot, a^{-}\right)} \\
-\mu \frac{q_{b}-q_{a}}{b-a} \frac{\widetilde{H}}{(\widetilde{H}+\bar{H}) \bar{H}}+\frac{2 q_{a}^{2}-q_{a} q_{b}-q_{b}^{2}}{3(\widetilde{H}+\bar{H})^{2}} \\
F_{4}\left(\widetilde{H}, \widetilde{h}, q, q_{b}, q_{a}\right):=-\frac{q_{b}^{2}}{2}\left(\frac{1}{(\widetilde{h}+\bar{h})^{2}\left(\cdot, b^{+}\right)}-\frac{1}{(\widetilde{H}+\bar{H})^{2}}\right)-\frac{\mu}{\bar{h}\left(\frac{\widetilde{h}}{(\widetilde{h}+\bar{h})} \frac{\partial q}{\partial x}\right)\left(\cdot, b^{+}\right)} \\
+\mu \frac{q_{b}-q_{a}}{b-a} \frac{\widetilde{H}}{(\widetilde{H}+\bar{H}) \bar{H}}-\frac{2 q_{b}^{2}-q_{a} q_{b}-q_{a}^{2}}{3(\widetilde{H}+\bar{H})^{2}}
\end{array}\right.
\end{array}
$$

Using (3.10), (3.11) and (3.12), we add the following initial conditions to system (6.5)-(6.14):

$$
\begin{array}{rlrl}
\widetilde{h}(0, x) & =\widetilde{h}_{0}(x) & (x \in \mathcal{E}), \\
q(0, x) & =q_{0}(x) & (x \in \mathcal{E}), \\
\widetilde{H}(0)=\widetilde{H}_{0}, & q_{a}(0) & =q_{a, 0} & q_{b}(0)=q_{b, 0},
\end{array}
$$

where

$$
\widetilde{H}_{0}=H_{0}-\bar{H}, \quad \widetilde{h}_{0}:=h_{0}-\bar{h}, \quad q_{a, 0}:=q_{0}(a), \quad q_{b, 0}:=q_{0}(b) .
$$

In order to study the above equations, we consider the following linear system

$$
\begin{array}{ccc}
\dot{\tilde{H}}=-\frac{q_{b}-q_{a}}{b-a} & (t \geqslant 0), \\
\frac{\partial \widetilde{h}}{\partial t}(t, x)+\frac{\partial q}{\partial x}(t, x)=0 & (t>0, x \in \mathcal{E}), \\
\frac{\partial q}{\partial t}+g \bar{h} \frac{\partial \widetilde{h}}{\partial x}-\mu \frac{\partial}{\partial x}\left(\frac{\partial q}{\partial x}\right)=f_{1} & (t>0, x \in \mathcal{E}), \\
q(t, 0)=q(t, \ell)=0 & (t>0), \\
q(t, a)=q_{a}(t), \quad q(t, b)=q_{b}(t) & (t>0), \\
{\left[\begin{array}{l}
\dot{q}_{a} \\
\dot{q}_{b}
\end{array}\right]=S_{0}(\bar{H})\left[\begin{array}{cc}
\frac{\mu}{\bar{H}} \frac{q(\cdot, b)-q(\cdot, a)}{b-a}+g\left(\widetilde{h}\left(\cdot, a^{-}\right)-\widetilde{H}\right)-\frac{\mu}{\bar{h}} \frac{\partial q}{\partial x}\left(\cdot, a^{-}\right) \\
-\frac{\mu}{\bar{H}} \frac{q(\cdot, b)-q(\cdot, a)}{b-a}-g\left(\widetilde{h}\left(\cdot, b^{+}\right)-\widetilde{H}\right)+\frac{\mu}{\bar{h}} \frac{\partial q}{\partial x}\left(\cdot, b^{+}\right)
\end{array}\right]+f_{2}}
\end{array}
$$

where

$$
S_{0}(H)=\frac{H}{(b-a)\left(M+\frac{(b-a)^{3}}{12 H}\right)}\left[\begin{array}{ll}
M+\frac{(b-a)^{3}}{3 H} & M-\frac{(b-a)^{3}}{6 H} \\
M-\frac{(b-a)^{3}}{6 H} & M+\frac{(b-a)^{3}}{3 H}
\end{array}\right],
$$

and where $f_{1}$ and $f_{2}$ are now given. 


\subsection{Study of the Linear Problem}

In this section we use the methodology developed in [15] in order to prove that the linear problem introduced above can be described by an analytic $C^{0}$ semigroup in an appropriate Hilbert space and then we show that this semigroup is exponentially stable. To this end we begin by defining the following spaces:

$$
\begin{gathered}
X=\left\{[H, h, q]^{\top} \in \mathbb{C} \times H^{1}(\mathcal{E}) \times L^{2}(\mathcal{E}) \mid \int_{\mathcal{E}} h_{0}(x) \mathrm{d} x+H_{0}(b-a)=0\right\}, \quad \mathcal{U}=\mathbb{C}^{2}, \\
Z=\left\{[H, h, q]^{\top} \in \mathbb{C} \times H^{1}(\mathcal{E}) \times H^{2}(\mathcal{E}) \mid \int_{\mathcal{E}} h_{0}(x) \mathrm{d} x+H_{0}(b-a)=0, q_{0}(0)=q_{0}(\ell)=0\right\} .
\end{gathered}
$$

We also introduce the following operators:

$$
\begin{gathered}
L: \mathcal{D}(L) \rightarrow X, \quad L\left[\begin{array}{l}
H \\
h \\
q
\end{array}\right]=\left[\begin{array}{c}
-\frac{q(b)-q(a)}{b-a} \\
-\frac{\mathrm{d} q}{\mathrm{~d} x} \\
-g \bar{h} \frac{\mathrm{d} h}{\mathrm{~d} x}+\mu \frac{\mathrm{d}^{2} q}{\mathrm{~d} x^{2}}
\end{array}\right], \quad \mathcal{D}(L)=Z, \\
G: \mathcal{D}(G) \rightarrow \mathcal{U}, \quad G\left[\begin{array}{c}
H \\
h \\
q
\end{array}\right]=\left[\begin{array}{l}
q(a) \\
q(b)
\end{array}\right], \quad \mathcal{D}(G)=Z,
\end{gathered}
$$

and

$$
A:=\left.L\right|_{\operatorname{Ker}(G)} .
$$

The above definition yields

$$
\mathcal{D}(A)=\mathbb{C} \times H^{1}(\mathcal{E}) \times\left(H^{2} \cap H_{0}^{1}\right)(\mathcal{E}) \quad \text { and } \quad A\left[\begin{array}{c}
H \\
h \\
q
\end{array}\right]=\left[\begin{array}{c}
0 \\
-\frac{\mathrm{d} q}{\mathrm{~d} x} \\
-g \bar{h} \frac{\mathrm{d} h}{\mathrm{~d} x}+\mu \frac{\mathrm{d}^{2} q}{\mathrm{~d} x^{2}}
\end{array}\right] .
$$

Lemma 6.1. The operator $A: \mathcal{D}(A) \rightarrow X$ generates an analytic semigroup.

Proof. It clearly suffices to prove that the operator $\widetilde{A}$ defined by

$$
\begin{gathered}
\mathcal{D}(\widetilde{A})=H^{1}(\mathcal{E}) \times\left(H^{2} \cap H_{0}^{1}\right)(\mathcal{E}), \\
\widetilde{A}\left[\begin{array}{l}
h \\
q
\end{array}\right]=\left[\begin{array}{c}
-\frac{\mathrm{d} q}{\mathrm{~d} x} \\
-g \bar{h} \frac{\mathrm{d} h}{\mathrm{~d} x}+\mu \frac{\mathrm{d}^{2} q}{\mathrm{~d} x^{2}}
\end{array}\right] \quad\left(\left[\begin{array}{l}
h \\
q
\end{array}\right] \in \mathcal{D}(\widetilde{A})\right),
\end{gathered}
$$

generates an analytic semigroup in $\widetilde{X}=H^{1}(\mathcal{E}) \times L^{2}(\mathcal{E})$. To this aim, noticing that the operator defined by

$$
P\left[\begin{array}{l}
h \\
q
\end{array}\right]=\left[\begin{array}{c}
0 \\
-g \bar{h} \frac{\mathrm{d} h}{\mathrm{~d} x}
\end{array}\right]
$$

is in $\mathcal{L}(\widetilde{X})$, it suffices to prove that the operator $A_{1}$ defined by

$$
\begin{gathered}
\mathcal{D}\left(A_{1}\right)=\mathcal{D}(\widetilde{A}) \\
A_{1}\left[\begin{array}{l}
h \\
q
\end{array}\right]=\left[\begin{array}{c}
-\frac{\mathrm{d} q}{\mathrm{~d} x} \\
\mu \frac{\mathrm{d}^{2} q}{\mathrm{~d} x^{2}}
\end{array}\right] \quad\left(\left[\begin{array}{l}
h \\
q
\end{array}\right] \in \mathcal{D}(\widetilde{A})\right),
\end{gathered}
$$


generates an analytic semigroup in $\tilde{X}$. Denoting

$$
\Sigma_{\varepsilon, \gamma}=\{\lambda \in \mathbb{C} \backslash\{0\}|| \arg \lambda|\leqslant \pi-\varepsilon, \quad| \lambda \mid>\gamma\},
$$

this is equivalent to the existence of $\varepsilon \in(0, \pi / 2)$ and $\gamma \geqslant 0$ such that

$$
\Sigma_{\varepsilon, \gamma} \subset \rho\left(A_{1}\right)
$$

and the set

$$
\left\{\lambda\left(\lambda I-A_{1}\right)^{-1} \mid \lambda \in \Sigma_{\varepsilon, \gamma}\right\}
$$

is bounded in $\mathcal{L}(\widetilde{X})$. To do this, for $\left[\begin{array}{l}f \\ g\end{array}\right] \in \widetilde{X}$ we notes that the equation

$$
\left(\lambda I-A_{1}\right)\left[\begin{array}{l}
h_{\lambda} \\
q_{\lambda}
\end{array}\right]=\left[\begin{array}{l}
f \\
g
\end{array}\right],
$$

writes

$$
\begin{gathered}
\lambda h_{\lambda}+\frac{d q_{\lambda}}{d x}=f, \\
\lambda q_{\lambda}-\mu \frac{\mathrm{d}^{2} q_{\lambda}}{\mathrm{d} x^{2}}=g, \\
q(0)=q(a)=q(b)=q(\ell)=0 .
\end{gathered}
$$

Classical results imply the existence of $\gamma, C \geqslant 0$ and $\varepsilon \in(0, \pi / 2)$ such that for every $\lambda \in \Sigma_{\varepsilon, \gamma}$ the unique solution of (6.33) and (6.34) satisfies

$$
\left\|\lambda q_{\lambda}\right\|_{L^{2}(\mathcal{E})}+\left\|q_{\lambda}\right\|_{H^{2}(\mathcal{E})} \leqslant C\|g\|_{L^{2}(\mathcal{E})} \quad\left(g \in L^{2}(\mathcal{E})\right) .
$$

Inserting the above estimate in (6.32) we obtain that

$$
\left\|\lambda h_{\lambda}\right\|_{H^{1}(\mathcal{E})} \leqslant\|f\|_{H^{1}(\mathcal{E})}+C\|g\|_{L^{2}(\mathcal{E})} \quad\left(f \in H^{1}(\mathcal{E}), g \in L^{2}(\mathcal{E})\right) .
$$

The last estimates and (6.35) imply that the family of operators

$$
\lambda\left(\lambda I-A_{1}\right)^{-1} \quad\left(\lambda \in \Sigma_{\varepsilon, \gamma}\right)
$$

is bounded in $\mathcal{L}(\widetilde{X})$, which ends the proof.

Using the notation

$$
z=\left[\begin{array}{c}
\widetilde{H} \\
\widetilde{h} \\
q
\end{array}\right], \quad u=\left[\begin{array}{l}
q_{a} \\
q_{b}
\end{array}\right],
$$

the linear system (6.18)-(6.24) writes

$$
\begin{gathered}
\dot{z}=L z+\left[\begin{array}{l}
0 \\
0 \\
f_{1}
\end{array}\right], \quad G z=u, \\
\dot{u}=\mathcal{C} z+f_{2},
\end{gathered}
$$

where $L$ and $G$ have been defined in (6.27) and (6.28), respectively, and

$$
\mathcal{C} z=S_{0}(\bar{H})\left[\begin{array}{c}
\frac{\mu}{\bar{H}} \frac{q(b)-q(a)}{b-a}+g\left(\widetilde{h}\left(a^{-}\right)-\widetilde{H}\right)-\frac{\mu}{\bar{h}} \frac{\mathrm{d} q}{\mathrm{~d} x}\left(a^{-}\right) \\
-\frac{\mu}{\bar{H}} \frac{q(b)-q(a)}{b-a}-g\left(\widetilde{h}\left(b^{+}\right)-\widetilde{H}\right)+\frac{\mu}{\bar{h}} \frac{\mathrm{d} q}{\mathrm{~d} x}\left(b^{+}\right)
\end{array}\right] .
$$


We introduce the operator $\mathcal{A}$ by:

$$
\mathcal{D}(\mathcal{A})=\left\{\left[\begin{array}{l}
z \\
u
\end{array}\right] \in Z \times \mathcal{U} ; G z=u\right\}
$$

and

$$
\mathcal{A}\left[\begin{array}{l}
z \\
u
\end{array}\right]=\left[\begin{array}{l}
L z \\
\mathcal{C} z
\end{array}\right]
$$

Then the linear system (6.18)-(6.24) can be written as

$$
\frac{d}{d t}\left[\begin{array}{l}
z \\
u
\end{array}\right]=\mathcal{A}\left[\begin{array}{l}
z \\
u
\end{array}\right]+\left[\begin{array}{l}
\Phi_{1} \\
\Phi_{2}
\end{array}\right]
$$

where

$$
\Phi_{1}=\left[\begin{array}{l}
0 \\
0 \\
f_{1}
\end{array}\right], \quad \Phi_{2}=f_{2} .
$$

Theorem 6.2. The operator $\mathcal{A}$ is the infinitesimal generator of an analytic semigroup on $X \times \mathcal{U}$.

Proof. From a trace theorem, one can check the following property on $\mathcal{C}$ :

$$
\forall \delta>0, \exists k(\delta) \quad\|\mathcal{C} z\|_{\mathbb{C}^{2}} \leqslant \delta\|z\|_{Z}+k(\delta)\|z\|_{X} .
$$

Then by [15, Theorem 1.25] and Lemma 6.1 we deduce that $\mathcal{A}: \mathcal{D}(\mathcal{A}) \rightarrow X \times \mathcal{U}$ generates an analytic semigroup.

We now show the following property on the resolvent set $\rho(\mathcal{A})$ of $\mathcal{A}$ :

Proposition 6.3. The resolvent set of $\mathcal{A}$ contains the closed right half plane of $\mathbb{C}$ :

$$
\mathbb{C}_{+}:=\{\lambda \in \mathbb{C} ; \operatorname{Re}(\lambda) \geqslant 0\} \subset \rho(\mathcal{A}) .
$$

Proof. We first show that $0 \in \rho(\mathcal{A})$, i.e. that $\mathcal{A}$ is boundedly invertible. Assume $F \in X \times \mathcal{U}$ and let us solve the equation

$$
\mathcal{A}\left[\begin{array}{l}
z_{0} \\
u_{0}
\end{array}\right]=F \quad\left(\left[\begin{array}{c}
z_{0} \\
u_{0}
\end{array}\right] \in \mathcal{D}(\mathcal{A})\right)
$$

Denoting $\left[\begin{array}{c}z_{0} \\ u_{0}\end{array}\right]=\left[\begin{array}{c}H_{0} \\ h_{0} \\ q_{0} \\ q_{a, 0} \\ q_{b, 0}\end{array}\right]$ and $F=\left[\begin{array}{c}f_{1} \\ f_{2} \\ f_{3} \\ f_{4} \\ f_{5}\end{array}\right]$, the above system writes

$$
\begin{gathered}
-\frac{q_{0}(b)-q_{0}(a)}{b-a}=f_{1}, \\
-\frac{\mathrm{d} q_{0}}{\mathrm{~d} x}(x)=f_{2}(x) \quad(x \in \mathcal{E}), \\
-g \bar{h} \frac{\mathrm{d} h_{0}}{\mathrm{~d} x}+\mu \frac{\mathrm{d}^{2} q_{0}}{\mathrm{~d} x^{2}}=f_{3}(x) \quad(x \in \mathcal{E}), \\
S_{0}(\bar{H})\left[\begin{array}{c}
\frac{\mu}{\bar{H}} \frac{q_{0}(b)-q_{0}(a)}{b-a}+g\left(h_{0}\left(a^{-}\right)-H_{0}\right)-\frac{\mu}{\bar{h}} \frac{\mathrm{d} q_{0}}{\mathrm{~d} x}\left(a^{-}\right) \\
-\frac{\mu}{\bar{H}} \frac{q_{0}(b)-q_{0}(a)}{b-a}-g\left(h_{0}\left(b^{+}\right)-H_{0}\right)+\frac{\mu}{\bar{h}} \frac{\mathrm{d} q_{0}}{\mathrm{~d} x}\left(b^{+}\right)
\end{array}\right]=\left[\begin{array}{l}
f_{4} \\
f_{5}
\end{array}\right]
\end{gathered}
$$




$$
\begin{gathered}
q_{0}(0)=q_{0}(\ell)=0, \\
q_{0}(a)=q_{a, 0}, \quad q_{0}(b)=q_{b, 0} .
\end{gathered}
$$

We first note that from (6.46) and (6.49) it follows that

$$
q_{0}(x)= \begin{cases}-\int_{0}^{x} f_{2}(\xi) \mathrm{d} \xi & \text { for } x \in[0, a] \\ \int_{x}^{\ell} f_{2}(\xi) \mathrm{d} \xi & \text { for } x \in[b, \ell] .\end{cases}
$$

Remark that since $\left[\begin{array}{l}f_{1} \\ f_{2} \\ f_{3}\end{array}\right] \in X$ (see (6.25)), equation (6.45) is also satisfied. Moreover, from (6.50) it follows that

$$
q_{a, 0}=-\int_{0}^{a} f_{2}(\xi) \mathrm{d} \xi, \quad q_{b, 0}=\int_{b}^{\ell} f_{2}(\xi) \mathrm{d} \xi
$$

Multiplying both sides of (6.48) by $S_{0}(\bar{H})^{-1}$ and using (6.45), (6.46), we obtain

$$
\left[\begin{array}{l}
g\left(h_{0}\left(a^{-}\right)-H_{0}\right) \\
g\left(h_{0}\left(b^{+}\right)-H_{0}\right)
\end{array}\right]=\left[\begin{array}{l}
\frac{\mu}{\bar{H}} f_{1}-\frac{\mu}{\bar{h}} f_{2}\left(a^{-}\right)+\left[M+\frac{(b-a)^{3}}{3 \bar{H}}\right] \frac{f_{4}}{(b-a)^{2}}-\left[M-\frac{(b-a)^{3}}{6 \bar{H}}\right] \frac{f_{5}}{(b-a)^{2}} \\
\frac{\mu}{\bar{H}} f_{1}-\frac{\mu}{\bar{h}} f_{2}\left(b^{+}\right)+\left[M-\frac{(b-a)^{3}}{6 \bar{H}}\right] \frac{f_{4}}{(b-a)^{2}}-\left[M+\frac{(b-a)^{3}}{3 \bar{H}}\right] \frac{f_{5}}{(b-a)^{2}}
\end{array}\right] .
$$

On the other hand, by combining (6.46) and (6.47) it follows that

$$
\frac{\mathrm{d}}{\mathrm{d} x}\left(h_{0}-H_{0}\right)(x)=-\frac{f_{3}(x)}{g \bar{h}}-\frac{\mu}{g \bar{h}} \frac{\mathrm{d} f_{2}}{\mathrm{~d} x}(x) \quad(x \in \mathcal{E}),
$$

so that, according to (6.53), we have

$$
\begin{aligned}
h_{0}(x)-H_{0}= & \frac{\mu}{g \bar{H}} f_{1}+\left[M+\frac{(b-a)^{3}}{3 \bar{H}}\right] \frac{f_{4}}{g(b-a)^{2}} \\
& -\left[M-\frac{(b-a)^{3}}{6 \bar{H}}\right] \frac{f_{5}}{g(b-a)^{2}}+\frac{1}{g \bar{h}} \int_{x}^{a} f_{3}(\xi) \mathrm{d} \xi-\frac{\mu}{g \bar{h}} f_{2}(x) \quad(x \in[0, a]), \\
h_{0}(x)-H_{0}= & \frac{\mu}{g \bar{H}} f_{1}+\left[M+\frac{(b-a)^{3}}{3 \bar{H}}\right] \frac{f_{4}}{g(b-a)^{2}} \\
& -\left[M-\frac{(b-a)^{3}}{6 \bar{H}}\right] \frac{f_{5}}{g(b-a)^{2}}-\frac{1}{g \bar{h}} \int_{b}^{x} f_{3}(\xi) \mathrm{d} \xi-\frac{\mu}{g \bar{h}} f_{2}(x) \quad(x \in[b, \ell]) .
\end{aligned}
$$

The above formulas, combined with the the fact that $\int_{\mathcal{E}}\left(h_{0}(x)-H_{0}\right) \mathrm{d} x+H_{0} \ell=0$, give an explicit formula for $H_{0}$ in terms of $f_{k}$, with $k \in\{1, \ldots, 5\}$, together with the existence of some positive constant $K_{0}$ such that

$$
\left|H_{0}\right| \leqslant K_{0}\|F\|_{X \times \mathcal{U}} \quad(F \in X \times \mathcal{U}) .
$$

The above estimate, together with (6.55) and (6.56) imply the existence of $K_{1}>0$ such that

$$
\left\|h_{0}\right\|_{L^{2}(\mathcal{E})} \leqslant K_{1}\|F\|_{X \times \mathcal{U}} \quad(F \in X \times \mathcal{U}) .
$$

The last two estimates and (6.54), (6.51), (6.52) imply that there exists $K_{2}>0$ such that

$$
\left\|\left[\begin{array}{l}
z_{0} \\
u_{0}
\end{array}\right]\right\|_{X \times \mathcal{U}} \leqslant K_{2}\|F\|_{X \times \mathcal{U}} \quad(F \in X \times \mathcal{U}) .
$$

This shows that $0 \in \rho(\mathcal{A})$. Assume now $\lambda \in \mathbb{C}_{+} \backslash\{0\}$ and $F=\left[f_{1}, f_{2}, f_{3}, f_{4}, f_{5}\right]^{\top}$, the equation

$$
(\lambda I-\mathcal{A})\left[\begin{array}{l}
z_{0} \\
u_{0}
\end{array}\right]=F
$$


writes

$$
\begin{gathered}
\lambda H_{0}+\frac{q_{0}(b)-q_{0}(a)}{b-a}=f_{1} \\
\lambda h_{0}(x)+\frac{\mathrm{d} q_{0}}{\mathrm{~d} x}(x)=f_{2}(x) \\
\lambda q_{0}(x)+g \bar{h} \frac{\mathrm{d} h_{0}}{\mathrm{~d} x}-\mu \frac{\mathrm{d}^{2} q_{0}}{\mathrm{~d} x^{2}}=f_{3}(x) \\
q_{0}(0)=q_{0}(\ell)=0, \\
\lambda\left[\begin{array}{c}
q_{a, 0} \\
q_{b, 0}
\end{array}\right]-S_{0}(\bar{H})\left[\begin{array}{c}
\frac{\mu}{\bar{H}} \frac{q_{0}(b)-q_{0}(a)}{b-a}+g\left(h_{0}\left(a^{-}\right)-H_{0}\right)-\frac{\mu}{\bar{h}} \frac{\mathrm{d} q_{0}}{\mathrm{~d} x}\left(a^{-}\right) \\
-\frac{\mu}{\bar{H}} \frac{q_{0}(b)-q_{0}(a)}{b-a}-g\left(h_{0}\left(b^{+}\right)-H_{0}\right)+\frac{\mu}{\bar{h}} \frac{\mathrm{d} q_{0}}{\mathrm{~d} x}\left(b^{+}\right)
\end{array}\right]=\left[\begin{array}{l}
f_{4} \\
f_{5}
\end{array}\right] .
\end{gathered}
$$

From (6.62) and (6.24), it follows that

$$
\begin{aligned}
& \frac{\mu}{\bar{H}} \frac{q_{0}(b)-q_{0}(a)}{b-a}+g\left(h_{0}\left(a^{-}\right)-H_{0}\right)-\frac{\mu}{\bar{h}} \frac{\mathrm{d} q_{0}}{\mathrm{~d} x}\left(a^{-}\right) \\
& =\left[M+\frac{(b-a)^{3}}{3 \bar{H}}\right] \frac{\left(\lambda q_{a, 0}-f_{4}\right)}{(b-a)^{2}}+\left[M-\frac{(b-a)^{3}}{6 \bar{H}}\right] \frac{\left(f_{5}-\lambda q_{b, 0}\right)}{(b-a)^{2}} \\
& -\frac{\mu}{\bar{H}} \frac{q_{0}(b)-q_{0}(a)}{b-a}-g\left(h_{0}\left(b^{+}\right)-H_{0}\right)+\frac{\mu}{\bar{h}} \frac{\mathrm{d} q_{0}}{\mathrm{~d} x}\left(b^{+}\right) \\
& =\left[M-\frac{(b-a)^{3}}{6 \bar{H}}\right] \frac{\left(f_{4}-\lambda q_{a, 0}\right)}{(b-a)^{2}}+\left[M+\frac{(b-a)^{3}}{3 \bar{H}}\right] \frac{\left(\lambda q_{b, 0}-f_{5}\right)}{(b-a)^{2}} .
\end{aligned}
$$

We next transform (6.57)-(6.64) into a boundary value problem for $q_{0}$ by eliminating $h_{0}, H_{0}, q_{a, 0}, q_{b, 0}$ from the above mentioned equations. Firstly, from (6.58) and (6.59) we deduce

$$
\frac{\lambda}{\bar{h}} q_{0}-\left(\frac{\mu}{\bar{h}}+\frac{g}{\lambda}\right) \frac{\mathrm{d}^{2} q_{0}}{\mathrm{~d} x^{2}}=\frac{f_{3}}{\bar{h}}-\frac{g}{\lambda} \frac{\mathrm{d} f_{2}}{\mathrm{~d} x}=: \phi_{1} \in L^{2}(\mathcal{E}) .
$$

Next, using (6.57), (6.58) and (6.61) in (6.63)-(6.64) it follows that

$$
\begin{aligned}
& \left(\frac{\mu}{\bar{h}}+\frac{g}{\lambda}\right) \frac{\mathrm{d} q_{0}}{\mathrm{~d} x}\left(a^{-}\right)=\left(\frac{\mu}{\bar{H}}+\frac{g}{\lambda}+\frac{\lambda M}{b-a}\right) \frac{q_{0}(b)-q_{0}(a)}{b-a}-\frac{\lambda(b-a)}{6 \bar{H}}\left(2 q_{0}(a)+q_{0}(b)\right)+\phi_{2}, \\
& \left(\frac{\mu}{\bar{h}}+\frac{g}{\lambda}\right) \frac{\mathrm{d} q_{0}}{\mathrm{~d} x}\left(b^{+}\right)=\left(\frac{\mu}{\bar{H}}+\frac{g}{\lambda}+\frac{\lambda M}{b-a}\right) \frac{q_{0}(b)-q_{0}(a)}{b-a}+\frac{\lambda(b-a)}{6 \bar{H}}\left(q_{0}(a)+2 q_{0}(b)\right)+\phi_{3},
\end{aligned}
$$

with

$$
\begin{aligned}
& \phi_{2}:=g\left(\frac{f_{2}\left(a^{-}\right)}{\lambda}-\frac{f_{1}}{\lambda}\right)+\left[M+\frac{(b-a)^{3}}{3 \bar{H}}\right] \frac{f_{4}}{(b-a)^{2}}-\left[M-\frac{(b-a)^{3}}{6 \bar{H}}\right] \frac{f_{5}}{(b-a)^{2}}, \\
& \phi_{3}:=g\left(\frac{f_{2}\left(b^{+}\right)}{\lambda}-\frac{f_{1}}{\lambda}\right)+\left[M-\frac{(b-a)^{3}}{6 \bar{H}}\right] \frac{f_{4}}{(b-a)^{2}}-\left[M+\frac{(b-a)^{3}}{3 \bar{H}}\right] \frac{f_{5}}{(b-a)^{2}} .
\end{aligned}
$$

At this stage we remark that the fact that $\mathbb{C}_{+} \backslash\{0\} \subset \rho(A)$ readily follows as soon as we have shown that for every $\lambda \in \mathbb{C}_{+} \backslash\{0\} \subset \rho(A)$ and $F \in X \times \mathcal{U}$, the system formed by (6.65) and (6.66)-(6.67), together with (6.60) admits a unique solution $q_{0}$ with

$$
\left\|q_{0}\right\|_{H^{2}(\mathcal{E})} \leqslant K(\lambda)\|F\|_{X \times \mathcal{U}} \quad(F \in X \times \mathcal{U}) .
$$


We then recover $h_{0}, H_{0}, q_{a, 0}, q_{b, 0}$ by using (6.57), (6.58) and (6.61).

Now, to solve (6.65), (6.60) and (6.66)-(6.67), it is sufficient to prove the existence and uniqueness of a weak solution. The $H^{2}$ regularity can then be obtained in a standard way.

Let us set

$$
V:=\left\{\psi \in H^{1}(\mathcal{E}) \mid \psi(0)=\psi(\ell)=0\right\} .
$$

Then we can check (6.65), (6.60) and (6.66)-(6.67) are equivalent to

$$
B_{\lambda}(q, \psi)=\int_{\mathcal{E}} \phi_{1} \bar{\psi} \mathrm{d} x+\phi_{2} \bar{\psi}(a)-\phi_{3} \bar{\psi}(b) \quad(\psi \in V),
$$

where

$$
\begin{aligned}
B_{\lambda}(q, \psi):= & \int_{\mathcal{E}}\left[\frac{\lambda}{\bar{h}} q \bar{\psi}+\left(\frac{\mu}{\bar{h}}+\frac{g}{\lambda}\right) \frac{\mathrm{d} q_{0}}{\mathrm{~d} x} \frac{\mathrm{d} \bar{\psi}}{\mathrm{d} x}\right] \mathrm{d} x \\
& -\left[\left(\frac{g}{\lambda}+\frac{\mu}{\bar{H}}+\frac{\lambda M}{b-a}\right) \frac{q_{0}(b)-q_{0}(a)}{b-a}-\frac{\lambda(b-a)}{6 \bar{H}}\left[2 q_{0}(a)+q_{0}(b)\right]\right] \bar{\psi}(a) \\
+ & {\left[\left(\frac{g}{\lambda}+\frac{\mu}{\bar{H}}+\frac{\lambda M}{b-a}\right) \frac{q_{0}(b)-q_{0}(a)}{b-a}+\frac{\lambda(b-a)}{6 \bar{H}}\left[q_{0}(a)+2 q_{0}(b)\right]\right] \bar{\psi}(b) \quad(q, \psi \in V) . }
\end{aligned}
$$

We notice that $B_{\lambda}$ writes more conveniently as

$$
\begin{aligned}
B_{\lambda}(q, \psi)=\int_{\mathcal{E}}\left[\frac{\lambda}{\bar{h}} q \bar{\psi}+\right. & \left.\left(\frac{\mu}{\bar{h}}+\frac{g}{\lambda}\right) \frac{\mathrm{d} q_{0}}{\mathrm{~d} x} \frac{\mathrm{d} \bar{\psi}}{\mathrm{d} x}\right] \mathrm{d} x \\
& +\left(\frac{g}{\lambda}+\frac{\mu}{\bar{H}}+\frac{\lambda M}{b-a}\right) \frac{\left(q_{0}(b)-q_{0}(a)\right) \overline{(\psi(b)-\psi(a)}}{b-a} \\
+ & \frac{\lambda(b-a)}{6 \bar{H}}\left[q_{0}(a) \bar{\psi}(a)+q_{0}(b) \bar{\psi}(b)+\left(q_{0}(b)+q_{0}(a)\right)(\bar{\psi}(b)+\bar{\psi}(a))\right] \quad(q, \psi \in V) .
\end{aligned}
$$

From the above formula and the Poincare inequality, it follows that for any $\lambda \in \mathbb{C}_{+} \backslash\{0\}$ there exist positive constants $C=C(\lambda), \alpha=\alpha(\lambda)$ such that

$$
\mid B_{\lambda}(q, \psi)\|\leqslant C\| q\left\|_{V}\right\| \psi\left\|_{V}, \quad \operatorname{Re} B_{\lambda}(q, q) \geqslant \alpha\right\| q \|_{V}^{2} \quad(q, \psi \in V),
$$

thus $B_{\lambda}$ is a bounded and coercive form on $V$. Moreover, the right hand side of (6.68) clearly defines a bounded linear functional on $V$. Thus the conclusion follows by the complex version of the Lax-Milgram Lemma (see, for instance, Arendt et al. [1, Lemma 5.4]).

Proposition 6.4. The operator $\mathcal{A}$ generates an exponentially stable semigroup $\left(e^{t \mathcal{A}}\right)_{t \geqslant 0}$ on $X \times \mathcal{U}$. In other words, there exist constant $C>0$ and $\eta_{0}>0$ such that

$$
\left\|e^{t \mathcal{A}}\left(\widetilde{H}_{0}, \widetilde{h}_{0}, q_{0}, q_{a, 0}, q_{b, 0}\right)^{\top}\right\|_{X \times \mathcal{U}} \leqslant C e^{-\eta_{0} t}\left\|\left(\widetilde{H}_{0}, \widetilde{h}_{0}, q_{0}, q_{a, 0}, q_{b, 0}\right)^{\top}\right\|_{X \times \mathcal{U}} .
$$

Proof. By Lemma 6.1 we have the existence of $C>0$ such that for any $\lambda \in \Sigma_{\varepsilon, \gamma}$ with $\varepsilon \in(0, \pi / 2)$,

$$
\left\|(\lambda I-\mathcal{A})^{-1}\right\|_{\mathcal{L}(X \times \mathcal{U})} \leqslant C .
$$

Next, by Proposition 6.3 and noting the fact that $\mathbb{C}_{+} \backslash \Sigma_{\varepsilon, \gamma}$ is a compact set, we obtain

$$
\left\|(\lambda I-\mathcal{A})^{-1}\right\|_{\mathcal{L}(X \times \mathcal{U})} \leqslant C, \text { for any } \lambda \in \mathbb{C}_{+} \cup \Sigma_{\varepsilon, \gamma}
$$

This yields that

$$
\{\lambda \in \mathbb{C} \mid \operatorname{Re} \lambda \geqslant-\eta\} \subset \rho(\mathcal{A}) .
$$

Finally applying Proposition 2.9 [2, p. 120] we deduce the exponential stability of the semigroup generated by the operator $\mathcal{A}$. 
We conclude from Theorem 6.2 and Proposition 6.4 the following result:

Proposition 6.5. Let $\eta \in\left[0, \eta_{0}\right)$ where $\eta_{0}$ is the constant introduced in Proposition 6.4. Then for any

$$
\left[\widetilde{H}_{0}, \widetilde{h}_{0}, q_{0}, q_{a, 0}, q_{b, 0}\right]^{\top} \in \mathbb{R} \times H^{1}(\mathcal{E}) \times H^{1}(\mathcal{E}) \times \mathbb{R}^{2}
$$

with

$$
\int_{\mathcal{E}} \widetilde{h}_{0}(x) \mathrm{d} x+\widetilde{H}_{0}(b-a)=0, \quad q_{0}(0)=q_{0}(\ell)=0, \quad q_{0}(a)=q_{a, 0}, \quad q_{0}(b)=q_{b, 0},
$$

and for any $e^{\eta(\cdot)} f_{1} \in L^{2}\left(0, \infty ; L^{2}(\mathcal{E})\right), e^{\eta(\cdot)} f_{2} \in L^{2}\left(0, \infty ; \mathbb{R}^{2}\right)$ the system $(6.18)-(6.23)$, with (6.15)(6.17) admits a unique solution

$$
\begin{gathered}
e^{\eta(\cdot)} \widetilde{H} \in H^{1}(0, \infty), \quad e^{\eta(\cdot)} \widetilde{h} \in H^{1}\left(0, \infty ; H^{1}(\mathcal{E})\right), \\
e^{\eta(\cdot)} q \in H^{1}\left(0, \infty ; L^{2}(\mathcal{E})\right) \cap C_{b}\left([0, \infty) ; H^{1}(\mathcal{E})\right) \cap L^{2}\left(0, \infty ; H^{2}(\mathcal{E})\right), \\
e^{\eta(\cdot)} q_{a} \in H^{1}(0, \infty), \quad e^{\eta(\cdot)} q_{b} \in H^{1}(0, \infty), \\
\int_{\mathcal{E}} \widetilde{h}(t, x) \mathrm{d} x+\widetilde{H}(t)(b-a)=0 \quad(t \geqslant 0) .
\end{gathered}
$$

Moreover, there exists a positive constant $C$ depending on $\eta$ such that

$$
\begin{aligned}
\left\|e^{\eta(\cdot)} \widetilde{H}\right\|_{H^{1}(0, \infty)}+ & \left\|e^{\eta(\cdot)} \widetilde{h}\right\|_{H^{1}\left(0, \infty ; H^{1}(\mathcal{E})\right)}+\left\|e^{\eta(\cdot)} q\right\|_{H^{1}\left(0, \infty ; L^{2}(\mathcal{E})\right) \cap C_{b}\left([0, \infty) ; H^{1}(\mathcal{E})\right) \cap L^{2}\left(0, \infty ; H^{2}(\mathcal{E})\right)} \\
& +\left\|e^{\eta(\cdot)}\left(q_{a}, q_{b}\right)\right\|_{H^{1}\left(0, \infty ; \mathbb{R}^{2}\right)} \\
\leqslant & \left(\left|\widetilde{H}_{0}\right|+\left\|\widetilde{h}_{0}\right\|_{H^{1}(\mathcal{E})}+\left\|q_{0}\right\|_{H^{1}(\mathcal{E})}+\left\|e^{\eta(\cdot)}\left(f_{1}, f_{2}\right)\right\|_{L^{2}\left(0, \infty ; L^{2}(\mathcal{E}) \times \mathbb{R}^{2}\right)}\right)
\end{aligned}
$$

\subsection{Fixed point}

Let us fix $\eta \in\left(0, \eta_{0}\right)$, where $\eta_{0}$ is the constant introduced in Proposition 6.4. We consider for all $\varepsilon>0$ the ball

$$
\mathfrak{B}_{\varepsilon}:=\left\{\left(f_{1}, f_{2}\right) \in L^{2}\left(0, \infty ; L^{2}(\mathcal{E}) \times \mathbb{R}^{2}\right) ;\left\|e^{\eta(\cdot)}\left(f_{1}, f_{2}\right)\right\|_{L^{2}\left(0, \infty ; L^{2}(\mathcal{E}) \times \mathbb{R}^{2}\right)} \leqslant \varepsilon\right\}
$$

and the map

$$
\Xi:\left(f_{1}, f_{2}\right) \in \mathfrak{B}_{\varepsilon} \mapsto\left(F_{1}(\widetilde{h}, q), F_{2}\left(\widetilde{H}, \widetilde{h}, q, q_{b}, q_{a}\right)\right)
$$

where $\left(\tilde{H}, \tilde{h}, q, q_{b}, q_{a}\right)$ is the solution of $(6.18)-(6.23)$, with $(6.15)-(6.17)$ associated with $\left(f_{1}, f_{2}\right)$, and where $F_{1}$ and $F_{2}$ are given by $(6.11)-(6.14)$. We take

$$
\left|\widetilde{H}_{0}\right|+\left\|\widetilde{h}_{0}\right\|_{H^{1}(\mathcal{E})}+\left\|q_{0}\right\|_{H^{1}(\mathcal{E})} \leqslant \varepsilon
$$

so that (6.76) yields

$$
\begin{array}{r}
\left\|e^{\eta(\cdot)} \tilde{H}\right\|_{H^{1}(0, \infty)}+\left\|e^{\eta(\cdot)} \widetilde{h}\right\|_{H^{1}\left(0, \infty ; H^{1}(\mathcal{E})\right)}+\left\|e^{\eta(\cdot)} q\right\|_{H^{1}\left(0, \infty ; L^{2}(\mathcal{E})\right) \cap C_{b}\left([0, \infty) ; H^{1}(\mathcal{E})\right) \cap L^{2}\left(0, \infty ; H^{2}(\mathcal{E})\right)} \\
+\left\|e^{\eta(\cdot)}\left(q_{a}, q_{b}\right)\right\|_{H^{1}\left(0, \infty ; \mathbb{R}^{2}\right)} \leqslant C \varepsilon
\end{array}
$$

Taking $\varepsilon$ small enough, we deduce from the above estimate that

$$
\widetilde{H} \geqslant-\frac{1}{2} \bar{H}, \quad \widetilde{h} \geqslant-\frac{1}{2} \bar{h} \quad((t, x) \in(0, \infty) \times \overline{\mathcal{E}}) .
$$


From the above estimate and from the the Sobolev embeddings $H^{1}(0, \infty) \subset L^{\infty}(0, \infty)$ and $H^{1}(\mathcal{E}) \subset$ $L^{\infty}(\mathcal{E})$, we deduce

$$
\begin{aligned}
& \left\|\frac{1}{\bar{h}+\widetilde{h}}\right\|_{L^{\infty}((0, \infty) \times \mathcal{E})}+\left\|\frac{1}{\bar{h}+\widetilde{h}}\right\|_{L^{\infty}\left(0, \infty ; H^{1}(\mathcal{E})\right)} \leqslant C, \\
& \left\|e^{\eta(\cdot)} q\right\|_{L^{\infty}((0, \infty) \times \mathcal{E})} \leqslant C \varepsilon, \quad\left\|e^{\eta(\cdot) h}\right\|_{L^{\infty}((0, \infty) \times \mathcal{E})} \leqslant C \varepsilon,
\end{aligned}
$$

for some constant $C$ indepedent of $\varepsilon$. For simplicity, we assume now that

$$
\varepsilon \leqslant 1 \text {. }
$$

We deduce from (6.11), (6.80) and the above estimate that

$$
\left\|e^{\eta(\cdot)} F_{1}(\widetilde{h}, q)\right\|_{L^{2}\left(0, \infty ; L^{2}(\mathcal{E})\right)} \leqslant C \varepsilon^{2} .
$$

From (6.80) and (6.82), we have

$$
\left\|\frac{e^{\eta(\cdot) \widetilde{h}}}{(\widetilde{h}+\bar{h})} \frac{\partial q}{\partial x}\right\|_{L^{2}\left(0, \infty ; H^{1}(\mathcal{E})\right)} \leqslant C \varepsilon^{2}
$$

and thus

$$
\left\|e^{\eta(\cdot)}\left[\begin{array}{l}
F_{3}\left(\widetilde{H}, \widetilde{h}, q, q_{b}, q_{a}\right) \\
F_{4}\left(\widetilde{H}, \widetilde{h}, q, q_{b}, q_{a}\right)
\end{array}\right]\right\|_{L^{2}\left(0, \infty ; \mathbb{R}^{2}\right)} \leqslant C \varepsilon^{2} .
$$

We also deduce from (6.80) that

$$
\left\|e^{\eta(\cdot)}\left[\begin{array}{l}
g\left(\widetilde{h}\left(\cdot, a^{-}\right)-\widetilde{H}\right)-\frac{\mu}{\bar{h}} \frac{\partial q}{\partial x}\left(\cdot, a^{-}\right)+\frac{\mu}{\bar{H}} \frac{q_{b}-q_{a}}{b-a} \\
-g\left(\widetilde{h}\left(\cdot, b^{+}\right)-\widetilde{H}\right)+\frac{\mu}{\bar{h}} \frac{\partial q}{\partial x}\left(\cdot, a^{-}\right)-\frac{\mu}{\bar{H}} \frac{q_{b}-q_{a}}{b-a}
\end{array}\right]\right\|_{L^{2}\left(0, \infty ; \mathbb{R}^{2}\right)} \leqslant C \varepsilon .
$$

From (4.12), (6.81) and (6.80), we have

$$
\|S(\widetilde{H}+\bar{H})\|_{L^{\infty}\left(0, \infty ; \mathbb{R}^{4}\right)} \leqslant C, \quad\|S(\widetilde{H}+\bar{H})-S(\bar{H})\|_{L^{\infty}\left(0, \infty ; \mathbb{R}^{4}\right)} \leqslant C \varepsilon .
$$

We obtain from (6.12) and from the above estimates

$$
\left\|e^{\eta(\cdot)} F_{2}\left(\widetilde{H}, \widetilde{h}, q, q_{b}, q_{a}\right)\right\|_{L^{2}\left(0, \infty ; \mathbb{R}^{2}\right)} \leqslant C \varepsilon^{2} .
$$

This shows that for small $\varepsilon, \Xi$ defined by (6.78) satisfies $\Xi\left(\mathfrak{B}_{\varepsilon}\right) \subset \mathfrak{B}_{\varepsilon}$. With similar calculations, we can also obtain that for small $\varepsilon, \Xi_{\mid \mathfrak{B}_{\varepsilon}}$ is a strict contraction. This gives the existence and uniqueness of a solution for system (4.17)-(4.22). We have now to come back to system (3.1)-(3.12). We see that (4.17) yields (3.1) in $\mathcal{I}$ and there exists a unique

$$
p \in \frac{M g}{|\mathcal{I}|}+L^{2}\left(0, \infty ; \mathcal{P}_{2}(\mathcal{I})\right)
$$

satisfying (4.4) and

$$
\int_{a}^{b} p(t, x) \mathrm{d} x=-M \ddot{H}(t)+M g=M \frac{\dot{q}_{b}-\dot{q}_{a}}{b-a}+M g .
$$

Using (4.5) and (4.6), we deduce that $p(t, a)$ and $p(t, b)$ satisfies (4.9) and (4.10). We deduce from this and from (4.22) that (3.7) and (3.8) hold.

This concludes the proof of Theorem 3.3. 


\section{Return to the Equilibrium}

The return to the equilibrium problem is a particular configuration of the floating structure problem, which consists in starting from a configuration where the solid is not at its equilibrium state and with water at rest. The motion of the solid is often described in the literature by a linear integro-differential equation, known as the Cummins equation, which has been obtained in [6], in the case of an inviscid fluid filling the whole space and assuming small waves amplitude. A similar equation taking in consideration some nonlinear effects has been derived in [14]. The classical linear Cummins equation, in the case of vertical displacements of a floating structure reads:

$$
\left(M+m_{\infty}\right) \ddot{H}(t)=\alpha H+K * \dot{H},
$$

where $H$ denotes the displacement of the structure from the equilibrium position, $M$ denotes the mass of the structure, $m_{\infty}$ is the added mass at infinite frequency, $\alpha$ is the hydrostatic stiffness and $K$ is the radiation force impulse response function (see, for instance, [6]). In this section, our aim is to derive a similar equation taking into consideration the viscosity of the fluid and possibly the presence of an exterior boundary for the fluid. To this aim, we use a linearized version of the system (3.1)-(3.12) as departure point.

Let $\bar{h}$ and $\bar{H}$ be the equilibrium height for the fluid and the solid respectively. Then we have

$$
\bar{h}=\bar{H}+\frac{M}{b-a},
$$

where $M$ is the mass of the solid (see (3.18)). For simplicity, let us assume that

$$
\bar{h}=1, \quad M=1, \quad g=1, \quad \bar{p}=\frac{1}{b-a} .
$$

The system (3.1)-(3.12), linearized around the trajectory $(H, h, q, p)=(\bar{h}, \bar{H}, 0, \bar{p})$, reads as

$$
\begin{aligned}
& \frac{\partial h}{\partial t}+\frac{\partial q}{\partial x}=0, \quad x \in \mathcal{E}, \\
& \frac{\partial q}{\partial t}+\frac{\partial h}{\partial x}-\mu \frac{\partial^{2} q}{\partial x^{2}}=0, \quad x \in \mathcal{E}, \\
& h\left(t, a^{-}\right)-\mu \frac{\partial q}{\partial x}\left(t, a^{-}\right)=p\left(t, a^{+}\right)+H(t)-\mu \frac{\partial q}{\partial x}\left(t, a^{+}\right) \\
& h\left(t, b^{+}\right)-\mu \frac{\partial q}{\partial x}\left(t, b^{+}\right)=p\left(t, b^{-}\right)+H(t)-\mu \frac{\partial q}{\partial x}\left(t, b^{-}\right) \\
& \frac{\partial H}{\partial t}+\frac{\partial q}{\partial x}=0, \quad x \in \mathcal{I}, \\
& \frac{\partial q}{\partial t}+\frac{\partial p}{\partial x}=0, \quad x \in \mathcal{I}, \\
& \ddot{H}(t)=\int_{a}^{b} p(t, x) d x .
\end{aligned}
$$

As we are interested in the return to the equilibrium problem we consider the above system with the following initial data

$$
h(0, x)=h_{0}, \quad q(0, x)=0, \quad H(0)=H_{0},
$$

where $h_{0}$ is a constant, $h_{0} \neq 0, H_{0} \neq 0$ and

$$
h_{0}|\mathcal{E}|+H_{0}(b-a)=0 .
$$

The last condition means that the volume of fluid is the same in the initial configuration and at equilibrium and it is necessary only in the case when the fluid is bounded. 
Remark 7.1. The system (7.2)-(7.8) is equivalent to the system (6.18)-(6.24). Therefore by Proposition 6.5, the system (7.2)-(7.9) admits a unique solution

$$
\begin{aligned}
& H \in H^{2}(0, \infty), \quad h \in H^{1}\left(0, \infty ; H^{1}(\mathcal{E})\right) \cap C_{b}^{1}\left([0, \infty) ; L^{2}(\mathcal{E})\right), \\
& q \in C_{b}\left([0, \infty) ; H^{1}(0, \ell)\right) \\
& q_{\mid \mathcal{E}} \in H^{1}\left(0, \infty ; L^{2}(\mathcal{E})\right) \cap C_{b}\left([0, \infty) ; H^{1}(\mathcal{E})\right) \cap L^{2}\left(0, \infty ; H^{2}(\mathcal{E})\right), \\
& q_{\mid \mathcal{I}} \in H^{1}\left(0, \infty ; \mathcal{P}_{1}(\mathcal{I})\right), \quad p_{\mid \mathcal{I}} \in L^{2}\left(0, \infty ; \mathcal{P}_{2}(\mathcal{I})\right) .
\end{aligned}
$$

Our aim is to show that, in the above configuration, we can eliminate $h, q$ and $p$ from the above system to obtain an integro-differential equation of Cummins type for $H$. To this aim, we first differentiate (7.7) with respect to $x$ and using (7.4) -(7.6) we have

$$
\left\{\begin{array}{l}
\frac{\partial^{2} p}{\partial x^{2}}=\ddot{H}(t), \quad x \in \mathcal{I} \\
p\left(t, a^{+}\right)=p_{a}(t):=h\left(t, a^{-}\right)-\mu \frac{\partial q}{\partial x}\left(t, a^{-}\right)-H(t)-\mu \dot{H}(t) \\
p\left(t, b^{-}\right)=p_{b}(t):=h\left(t, b^{+}\right)-\mu \frac{\partial q}{\partial x}\left(t, b^{+}\right)-H(t)-\mu \dot{H}(t) .
\end{array}\right.
$$

We decompose $p$ as

$$
p=p_{1}+p_{2},
$$

where $p_{1}$ solves

$$
\frac{\partial^{2} p_{1}}{\partial x^{2}}=\ddot{H}(t), \quad p_{1}(t, a)=p_{1}(t, b)=0,
$$

and $p_{2}$ solves

$$
\frac{\partial^{2} p_{2}}{\partial x^{2}}=0, \quad p_{2}(t, a)=p_{a}(t), \quad p_{2}(t, b)=p_{b}(t)
$$

Therefore

$$
p_{1}(t, x)=\ddot{H}(t)\left(\frac{x^{2}}{2}-\frac{b+a}{2} x+\frac{a b}{2}\right)
$$

and

$$
p_{2}(t, x)=p_{a}(t)+\left(p_{b}(t)-p_{a}(t)\right) \frac{x-a}{b-a} .
$$

Substituting the values of $p_{1}$ and $p_{2}$ in (7.8), we obtain the following equation

$$
\left(1+\frac{1}{12}(b-a)^{3}\right) \ddot{H}(t)=p_{a}(t)(b-a)+\left(p_{b}(t)-p_{a}(t)\right) \frac{b-a}{2}=\frac{b-a}{2}\left(p_{a}(t)+p_{b}(t)\right) .
$$

Using the expression of $p_{a}$ and $p_{b}$ from (7.11) we can rewrite the above equation as

$$
\begin{aligned}
\left(1+\frac{1}{12}(b-a)^{3}\right) \ddot{H}(t)=\frac{b-a}{2}\left(h\left(t, a^{-}\right)-\mu \frac{\partial q}{\partial x}\left(t, a^{-}\right)+h\left(t, b^{+}\right)-\mu \frac{\partial q}{\partial x}\left(t, b^{+}\right)\right) \\
-(b-a)(H(t)+\mu \dot{H}(t)) .
\end{aligned}
$$

To simplify we assume symmetry of the fluid-structure configuration, i.e., we suppose that the solid is symmetric around the axis $x=x_{0}$ where $x_{0}=\frac{1}{2}(b+a)$ and that $\ell=a+b$. Thus we have

$$
h\left(t, a^{-}\right)=h\left(t, b^{+}\right) \quad q\left(t, b^{+}\right)=-q\left(t, a^{-}\right) .
$$

Then by taking the boundary trace of all the terms in (7.2), we obtain

$$
\frac{\partial q}{\partial x}\left(t, a^{-}\right)=\frac{\partial q}{\partial x}\left(t, b^{+}\right) .
$$


Therefore, (7.17) can be written as

$$
\left.\left(1+\frac{1}{12}(b-a)^{3}\right) \ddot{H}(t)=(b-a)\left(h\left(t, a^{-}\right)-\mu \frac{\partial q}{\partial x}\left(t, a^{-}\right)\right)\right)-(b-a)(H(t)+\mu \dot{H}(t)) .
$$

We now need to express $h\left(t, a^{-}\right)-\mu \frac{\partial q}{\partial x}\left(t, a^{-}\right)$in terms of $H$ and $\dot{H}$. For $x \in \mathcal{I}$ we have

$$
q(t, b)-q(t, a)=-(b-a) \dot{H}(t) .
$$

Using (7.18), we obtain

$$
q(t, a)=\frac{b-a}{2} \dot{H}(t), \quad q(t, b)=-\frac{b-a}{2} \dot{H}(t) .
$$

From (7.2)-(7.3), $q$ satisfy the following equation for $x \in[0, a]$

$$
\frac{\partial^{2} q}{\partial t^{2}}-\frac{\partial^{2} q}{\partial x^{2}}-\mu \frac{\partial^{3} q}{\partial t \partial x^{2}}=0, \quad q(t, 0)=0, \quad q(t, a)=\frac{b-a}{2} \dot{H}(t), \quad q(0, x)=\frac{\partial q}{\partial t}(0, x)=0
$$

For $f \in L^{1}[0, \infty)$ let $\widehat{f}$ be the Laplace transform of $f$. Applying this transform to both sides of (7.22) we obtain

$$
s^{2} \widehat{q}-(1+s \mu) \frac{\partial^{2} \widehat{q}}{\partial x^{2}}=0, \quad \widehat{q}(s, 0)=0, \quad \widehat{q}(s, a)=\frac{b-a}{2} \widehat{\dot{H}}, \quad \operatorname{Re}(s)>0 .
$$

Therefore

$$
\widehat{q}(s, x)=A(s) e^{-\frac{s}{\sqrt{1+s \mu}} x}+B(s) e^{\frac{s}{\sqrt{1+s \mu}} x},
$$

where $z \mapsto \sqrt{z}$ is the principal branch of the square root function.

Using the boundary conditions (7.23) it follows that

$$
A(s)+B(s)=0, \quad A(s) e^{-\frac{s a}{\sqrt{1+s \mu}}}+B(s) e^{\frac{s a}{\sqrt{1+s \mu}}}=\frac{b-a}{2} \hat{\dot{H}}
$$

Therefore, (7.24) yields

$$
\widehat{q}(s, x)=\frac{b-a}{2} \frac{e^{\frac{-s x}{\sqrt{1+s \mu}}}-e^{\frac{s x}{\sqrt{1+s \mu}}}}{e^{\frac{-s a}{\sqrt{1+s \mu}}}-e^{\frac{s a}{\sqrt{1+s \mu}}}} \hat{\dot{H}}(s) \quad(x \in[0, a], \quad \text { Re } s>0) .
$$

From the above formula it follows that

$$
\frac{\partial \widehat{q}}{\partial x}(s, a)=-\frac{b-a}{2} \widehat{f}_{a}(s) \widehat{\dot{H}}(s) \quad(\operatorname{Re} s>0),
$$

where

$$
\widehat{f}(s)=\frac{s}{\sqrt{1+s \mu}} \frac{e^{\frac{-s a}{\sqrt{1+s \mu}}}+e^{\frac{s a}{\sqrt{1+s \mu}}}}{e^{\frac{-s a}{\sqrt{1+s \mu}}}-e^{\frac{s a}{\sqrt{1+s \mu}}}} \quad(\operatorname{Re} s>0) .
$$

On the other hand, taking Laplace transform of (7.2) we have

$$
\widehat{h}(s, a)=\frac{h_{0}}{s}-\frac{1}{s} \frac{\partial \widehat{q}}{\partial x}(s, a) .
$$

Therefore,

$$
\widehat{h}(s, a)-\mu \frac{\partial \widehat{q}}{\partial x}(s, a)=\frac{h_{0}}{s}-\frac{b-a}{2} \widehat{F}(s) \widehat{\dot{H}}(s) \quad \text { with } \quad \widehat{F}(s)=-\left(\frac{1}{s}+\mu\right) \widehat{f}_{a}(s) .
$$


Let $F(t)$ denotes the inverse Laplace transform of $\widehat{F}(s)$. By taking the inverse Laplace transform in the above identity we obtain

$$
h\left(t, a^{-}\right)-\mu \frac{\partial q}{\partial x}\left(t, a^{-}\right)=h_{0}-\frac{b-a}{2} F * \dot{H} .
$$

Using the above expressions in (7.19) we have

$$
\left(1+\frac{1}{12}(b-a)^{3}\right) \ddot{H}=(b-a) h_{0}-\frac{(b-a)^{2}}{2} F * \dot{H}-(b-a) H-\mu(b-a) \dot{H} .
$$

Therefore, we have proved the following proposition

Proposition 7.2. Assume that $\ell=a+b$, i.e.that the solid is symmetric around the axis $x=x_{0}$, $x_{0}=\frac{1}{2}(a+b)$. Then, for the return to the equilibrium problem (7.2)-(7.10), the position of the solid is completely determined by the integro-differential equation

$$
\left\{\begin{array}{l}
\left(1+\frac{1}{12}(b-a)^{3}\right) \ddot{H}=-H_{0} \frac{|\mathcal{I}|^{2}}{|\mathcal{E}|}-\frac{(b-a)^{2}}{2} F * \dot{H}-(b-a) H-\mu(b-a) \dot{H}, \\
H(0)=H_{0}, \quad \dot{H}(0)=0
\end{array}\right.
$$

and with $F$ such that

$$
\widehat{F}(s)=-\sqrt{1+s \mu} \frac{e^{\frac{-s a}{\sqrt{1+s \mu}}}+e^{\frac{s a}{\sqrt{1+s \mu}}}}{e^{\frac{-s a}{\sqrt{1+s \mu}}}-e^{\frac{s a}{\sqrt{1+s \mu}}}} .
$$

The function $\widehat{F}$ growths like $\sqrt{\operatorname{Re} s}$ when $\operatorname{Re} s \rightarrow \infty$, thus $F$ is a distribution. Consequently, the second term in the right hand side of the first equation in (7.30) involves fractional derivatives of $H$, as explained with more details below. More precisely, in the remaining part of this section we consider the case in which the fluid domain is unbounded and we derive a generalized Cummins equation, taking in consideration the viscosity.

Let $\mathcal{E}=(-\infty, a) \cup(b, \infty)$ and take the initial data (for the linearized problem (7.2)-(7.9))

$$
h_{0}=0, \quad q_{0}=0, \quad H_{0} \neq 0 .
$$

In this case, for $x \in(-\infty, a)$ we have the following equation satisfied by $\widehat{q}(s, x)$ (instead of $(7.23)$ ):

$$
s^{2} \widehat{q}-(1+s \mu) \frac{\partial^{2} \widehat{q}}{\partial x^{2}}=0, \quad \widehat{q}(s, x) \rightarrow 0 \text { as } x \rightarrow-\infty, \quad \widehat{q}(s, a)=\frac{b-a}{2} \hat{\dot{H}}, \quad \operatorname{Re}(s)>0 .
$$

Then

$$
\widehat{q}(s, x)=\frac{b-a}{2} e^{\frac{-s a}{\sqrt{1+s \mu}}} e^{\frac{s x}{\sqrt{1+s \mu}}} \widehat{\dot{H}}(s),
$$

and

$$
\widehat{h}\left(s, a^{-}\right)-\mu \frac{\partial \widehat{q}}{\partial x}\left(s, a^{-}\right)=-\frac{b-a}{2}\left(\frac{1}{s}+\mu\right) \frac{s}{\sqrt{1+s \mu}} \widehat{\dot{H}}(s)=-\frac{b-a}{2}(\sqrt{1+\mu s}) \widehat{\dot{H}}(s) .
$$

In a similar manner we obtain

$$
\widehat{h}\left(s, b^{+}\right)-\mu \frac{\partial \widehat{q}}{\partial x}\left(s, b^{+}\right)=-\frac{b-a}{2}(\sqrt{1+\mu s}) \widehat{\dot{H}}(s) .
$$

Inserting the above expressions in (7.19), the equation for $H$ reads as

$$
\left(1+\frac{1}{12}(b-a)^{3}\right) \ddot{H}=-(b-a)^{2} F * \dot{H}-(b-a) H-\mu(b-a) \dot{H},
$$


where

$$
\widehat{F}(s)=\sqrt{1+\mu s} .
$$

Equation (7.36) can be further transformed in an equation involving a fractional derivative. Indeed, (7.37) can be rewritten

$$
\widehat{F}(s)=\widehat{F}_{1}(s)+\widehat{F}_{2}(s),
$$

where

$$
\begin{gathered}
\widehat{F}_{1}(s)=\sqrt{\mu s} \\
\widehat{F}_{2}(s)=\sqrt{\mu}\left(\sqrt{s+\frac{1}{\mu}}-\sqrt{s}\right)=\frac{1}{\sqrt{\mu}\left(\sqrt{s+\frac{1}{\mu}}+\sqrt{s}\right)} .
\end{gathered}
$$

Using tables of Laplace transforms we see that

$$
F_{2}(t)=\sqrt{\mu} \frac{1-\mathrm{e}^{-\frac{t}{\mu}}}{2 \sqrt{\pi t^{3}}} .
$$

On the other hand, (7.39) implies that $F_{1}$ is (up to a multiplicative constant) the fractional derivative of order $\frac{1}{2}$ of the Dirac mass at $t=0$, that is

$$
F_{1}=\sqrt{\mu} D^{\frac{1}{2}} \delta_{0} .
$$

Combining (7.36)-(7.42) we obtain the Cummins type equation

$$
\left(1+\frac{1}{12}(b-a)^{3}\right) \ddot{H}=-\frac{(b-a)^{2}}{2} F_{2} * \dot{H}-\sqrt{\mu}\left(D^{\frac{3}{2}} H\right)-(b-a) H-\mu(b-a) \dot{H} .
$$

Thus the outcome of the viscosity in this model is the presence of the standard damping term $\mu(b-a) \dot{H}$ and in the fractional damping term $\sqrt{\mu}\left(D^{\frac{3}{2}} H\right)$.

Remark 7.3. If $\mu=0$, then (7.36) becomes

$$
\left(1+\frac{1}{12}(b-a)^{3}\right) \ddot{H}(t)=-\frac{(b-a)^{2}}{2} \dot{H}-(b-a) H,
$$

which is the same as Eq. (57) of [14]. 


\section{Numerical Simulations}

In this section, we discuss some numerical simulations for the system nonlinear (4.17)-(4.22) and we compare with the results obtained via linearization. To this aim, consider some subdivisions of the intervals $[0, a]$ and $[b, \ell]$, and we use them to discretize the equations with respect to the space variable. Since the procedure is similar for the two intervals, we only present the method for the interval $[0, a]$. To simplify, we also assume that the subdivision $\left(x_{k}\right)$ of $[0, a]$ is uniform:

$$
x_{k}=\frac{k}{n} a, \quad k \in\{0, \ldots, n\}, \quad \delta x=\frac{a}{n} .
$$

We also consider the subdivision $\left(\widetilde{x}_{k}\right)$ :

$$
\widetilde{x}_{k}=\frac{k-1 / 2}{n} a, \quad k \in\{1, \ldots, n\} .
$$

Our aim is compute approximations of $h$ and $q$ respectively on $\left(\widetilde{x}_{k}\right)$ and on $\left(x_{k}\right)$ :

$$
\widetilde{h}_{k} \approx h\left(\cdot, \widetilde{x}_{k}\right) \quad q_{k} \approx q\left(\cdot, x_{k}\right) .
$$

In particular, $q_{0}=0$ and $q_{n} \approx q_{a}$ where $q_{a}$ is one of the unknowns of (4.17)-(4.22). With the above notation, it is natural to approximate the derivative of $q$ by the following formula:

$$
\left(\frac{\widetilde{\partial q}}{\partial x}\right)_{k}=\frac{q_{k}-q_{k-1}}{\delta x}, \quad k \in\{1, \ldots, n\}
$$

and (4.18) is approximated by

$$
\frac{d}{d t} \widetilde{h}_{k}=-\frac{q_{k}-q_{k-1}}{\delta x}, \quad k \in\{1, \ldots, n\} .
$$

In a similar way, (4.19) is approximated by

$$
\frac{d}{d t} q_{k}=-\frac{\widetilde{F}_{k+1}-\widetilde{F}_{k}}{\delta x}+h_{k} \frac{\left(\frac{\partial q}{\partial x}\right)_{k+1}-\left(\frac{\widetilde{\partial q}}{\partial x}\right)_{k}}{\delta x}, \quad k \in\{1, \ldots, n-1\},
$$

where

$$
\widetilde{F}_{k}=\frac{\widetilde{q}_{k}^{2}}{\widetilde{h}_{k}}+\frac{g \widetilde{h}_{k}^{2}}{2} .
$$

In the above formulas, we see that we need to compute $\widetilde{q}_{k}$ and $h_{k}$. For the height of fluid, we consider the formula

$$
h_{k}=\frac{\widetilde{h}_{k+1}+\widetilde{h}_{k}}{2}, \quad k \in\{1, \ldots, n-1\},
$$

and for $q$ we use an upwind approximation:

$$
\widetilde{q}_{k}=\frac{q_{k+1}+q_{k}}{2} \quad \text { or } \quad \frac{q_{k}+q_{k-1}}{2}, \quad k \in\{1, \ldots, n-1\},
$$

according to the sign of $\frac{q_{k+1}+q_{k}}{2}$.

Note that in (8.1), we don't need to compute the derivative of $q_{k}$ for $k=0\left(q_{0}=0\right)$ or for $k=n$ since $q_{n}$ corresponds to $q_{a}$ and its derivative is obtained through the equation (4.22).

Using the above numerical scheme, we can consider the return to the equilibrium problem, in its linear and nonlinear version. We place ourselves in the configuration described in Section 7. In particular, we take the initial conditions (7.9), with different values of $h_{0}, H_{0}$ and $\mu$ (see Figure 2 and Figure 3). 
We notice in both experiments that the convergence to the equilibrium is depending a lot of the viscosity. When the viscosity is large, both linear and nonlinear problem converges to the equilibrium very quickly. For instance, if $\mu=50$ we see that at $t=100$ the solutions are close to the equilibrium. On the contrary, when the viscosity is small $(\mu=5$ or 0.5$)$ trajectories do not converge to the equilibrium as fast as the previous one. Moreover, these simulations seem to support the conjecture that the return to equilibrium takes place without imposing initial data close to equilibrium.

We also see that the differences between the nonlinear problem and the linear problem are stronger for small viscosities, and we remark that, at least for these numerical tests, the nonlinear system goes faster than the linear system to the equilibrium. 

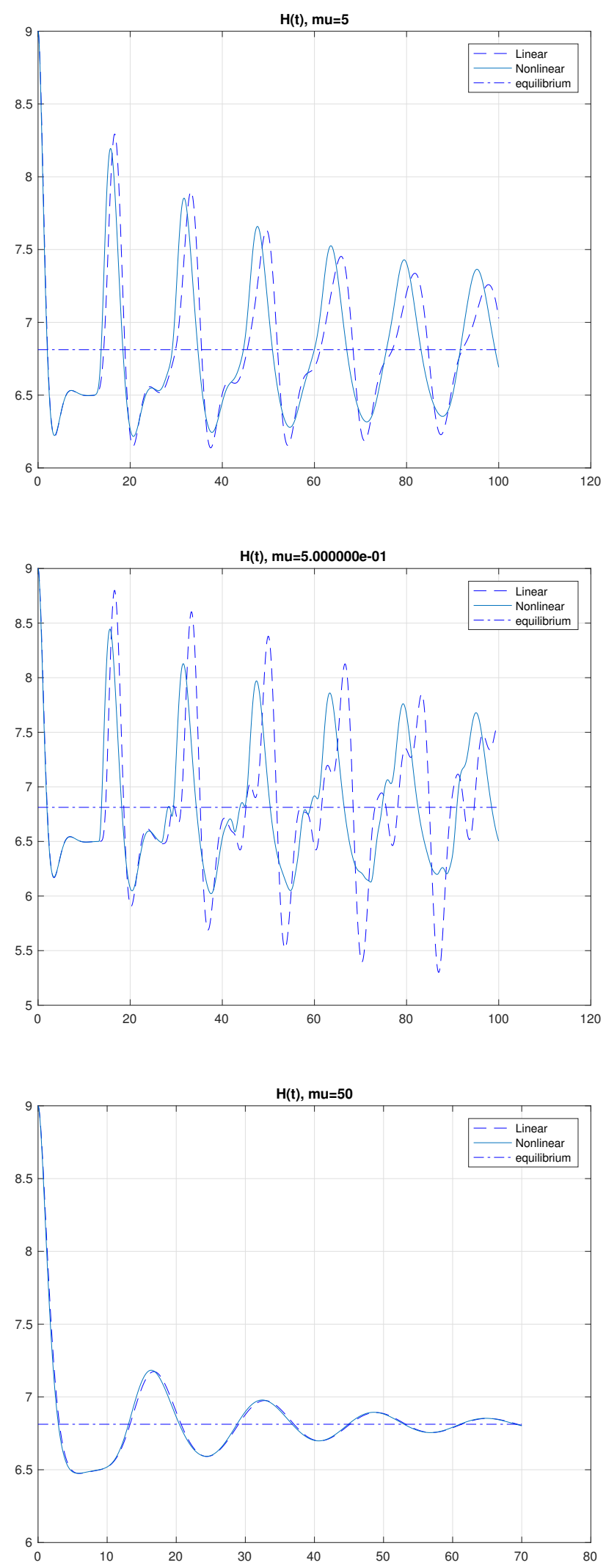

Figure 2: $H_{0}=9, \mu=5,0.5,50$ 

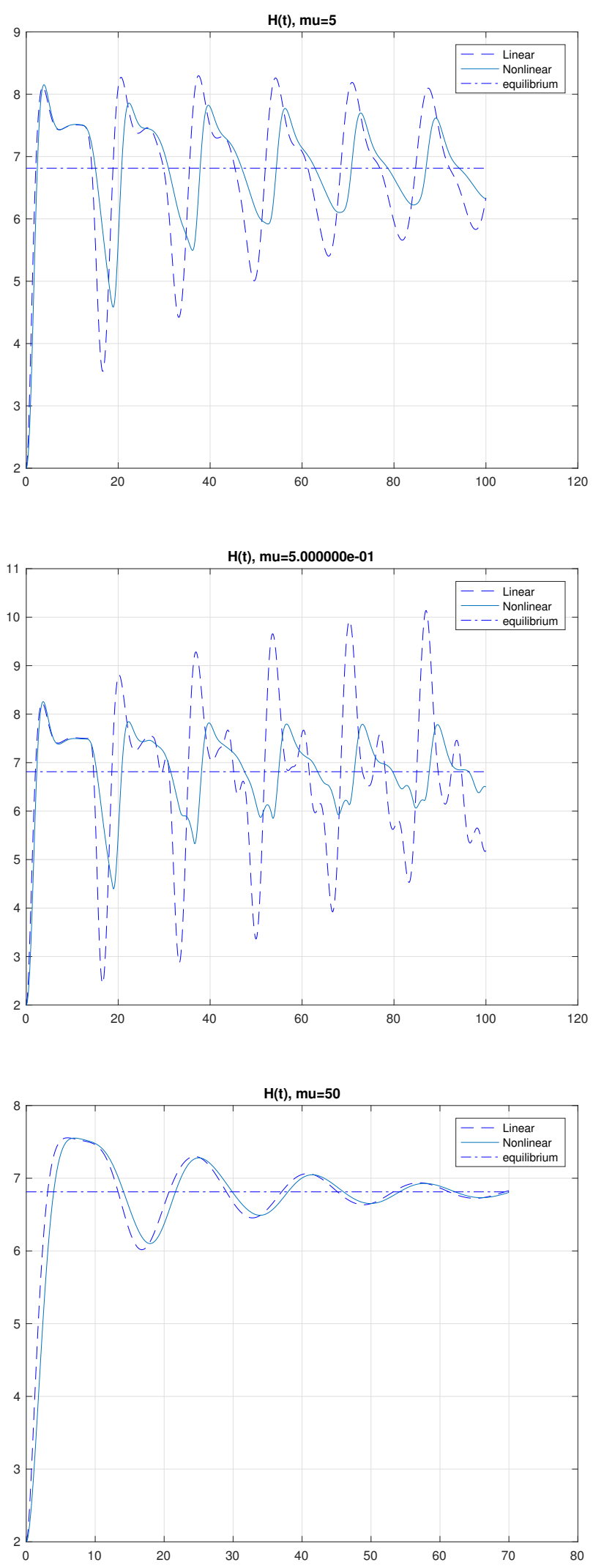

Figure 3: $H_{0}=2, \mu=5,0.5,50$ 


\section{References}

[1] W. Arendt, R. Chill, C. Seifert, H. Vogt, and J. Voigt, Form methods for evolution equations, and applications, in Lecture Notes of the 18th Internet Seminar on Evolution Equations, vol. 15, 2014.

[2] A. Bensoussan, G. Da Prato, M. C. Delfour, and S. K. Mitter, Representation and control of infinite dimensional systems, Systems \& Control: Foundations \& Applications, Birkhäuser Boston, Inc., Boston, MA, second ed., 2007.

[3] C. Bernardi and O. Pironneau, On the shallow water equations at low Reynolds number, Comm. Partial Differential Equations, 16 (1991), pp. 59-104.

[4] T. Bodnár, G. P. Galdi, And v. Nečasová, eds., Particles in flows, Advances in Mathematical Fluid Mechanics, Birkhäuser/Springer, Cham, 2017.

[5] D. Bresch, Shallow-water equations and related topics, in Handbook of differential equations: evolutionary equations. Vol. V, Handb. Differ. Equ., Elsevier/North-Holland, Amsterdam, 2009, pp. 1-104.

[6] W. Cummins, The impulse response function and ship motions, tech. rep., David Taylor Model Basin Washington DC, 1962.

[7] J. FALnes, Ocean waves and oscillating systems: linear interactions including wave-energy extraction, Cambridge university press, 2002.

[8] F. Gay-Balmaz and H. Yoshimura, A free energy lagrangian variational formulation of the Navier-Stokes-Fourier system, arXiv preprint arXiv:1706.09010, (2017).

[9] J.-F. Gerbeau And B. Perthame, Derivation of viscous Saint-Venant system for laminar shallow water; numerical validation, Discrete Contin. Dyn. Syst. Ser. B, 1 (2001), pp. 89-102.

[10] B. H. HaAk, D. Maity, T. Takahashi, and M. Tucsnak, Mathematical analysis of the motion of a rigid body in a compressible Navier-Stokes-Fourier fluid. working paper or preprint, Oct. 2017.

[11] F. John, On the motion of floating bodies. I, Comm. Pure Appl. Math., 2 (1949), pp. 13-57.

[12] — On the motion of floating bodies. II. Simple harmonic motions, Comm. Pure Appl. Math., 3 (1950), pp. 45-101.

[13] P. E. Kloeden, Global existence of classical solutions in the dissipative shallow water equations, SIAM J. Math. Anal., 16 (1985), pp. 301-315.

[14] D. Lannes, On the dynamics of floating structures, Ann. PDE, 3 (2017), pp. Art. 11, 81.

[15] D. Maity And M. Tucsnak, A maximal regularity approach to the analysis of some particulate flows, in Particles in flows, Adv. Math. Fluid Mech., Birkhäuser/Springer, Cham, 2017, pp. 1-75.

[16] P. Orenga, Un théorème d'existence de solutions d'un problème de shallow water, Arch. Rational Mech. Anal., 130 (1995), pp. 183-204.

[17] N. Petit And P. Rouchon, Dynamics and solutions to some control problems for water-tank systems, IEEE Transactions on Automatic Control, 47 (2002), pp. 594-609.

[18] J. M. Rodríguez And R. TABoAda-VÁzquez, From Navier-Stokes equations to shallow waters with viscosity by asymptotic analysis, Asymptot. Anal., 43 (2005), pp. 267-285.

[19] L. Sundbye, Global existence for the Dirichlet problem for the viscous shallow water equations, J. Math. Anal. Appl., 202 (1996), pp. 236-258. 